\title{
MVTN: Multi-View Transformation Network for 3D Shape Recognition
}

\author{
Abdullah Hamdi Silvio Giancola Bernard Ghanem \\ King Abdullah University of Science and Technology (KAUST), Thuwal, Saudi Arabia \\ \{abdullah.hamdi, silvio.giancola, bernard.ghanem\}@kaust.edu.sa
}

\begin{abstract}
Multi-view projection methods have demonstrated their ability to reach state-of-the-art performance on $3 D$ shape recognition. Those methods learn different ways to aggregate information from multiple views. However, the camera view-points for those views tend to be heuristically set and fixed for all shapes. To circumvent the lack of dynamism of current multi-view methods, we propose to learn those viewpoints. In particular, we introduce the Multi-View Transformation Network (MVTN) that regresses optimal view-points for $3 D$ shape recognition, building upon advances in differentiable rendering. As a result, MVTN can be trained end-to-end along with any multi-view network for $3 D$ shape classification. We integrate MVTN in a novel adaptive multiview pipeline that can render either $3 D$ meshes or point clouds. MVTN exhibits clear performance gains in the tasks of $3 D$ shape classification and $3 D$ shape retrieval without the need for extra training supervision. In these tasks, MVTN achieves state-of-the-art performance on ModelNet40, ShapeNet Core55, and the most recent and realistic ScanObjectNN dataset (up to 6\% improvement). Interestingly, we also show that MVTN can provide network robustness against rotation and occlusion in the $3 D$ domain. The code is available at https://github.com/a jhamdi/MVTN.
\end{abstract}

\section{Introduction}

Given its success in the 2D realm, deep learning naturally expanded to the $3 \mathrm{D}$ vision domain. In $3 \mathrm{D}$, deep networks achieve impressive results in classification, segmentation, and detection. 3D deep learning pipelines operate directly on 3D data, commonly represented as point clouds [62, 65, 74], meshes [19, 30], or voxels [59, 14, 25]. However, other methods choose to represent $3 \mathrm{D}$ information by rendering multiple 2D views of objects or scenes [69]. Such multi-view methods are more similar to a human approach, where the human visual system is fed with streams of rendered images instead of more elaborate 3D representations.

Recent developments in multi-view methods show im-
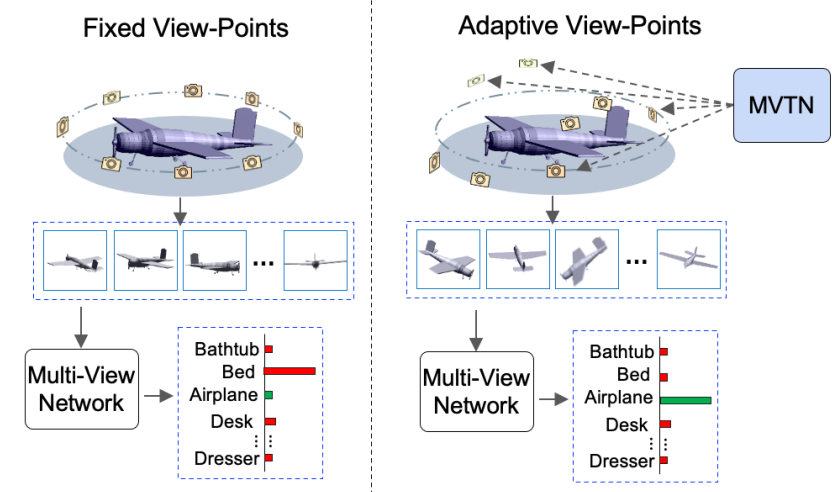

Figure 1. Multi-View Transformation Network (MVTN). We propose a differentiable module that predicts the best view-points for a task-specific multi-view network. MVTN is trained jointly with this network without any extra training supervision, while improving the performance on 3D classification and shape retrieval.

pressive performance, and in many instances, achieve stateof-the-art results in 3D shape classification and segmentation $[40,75,45,39,16]$. Multi-view approaches bridge the gap between $2 \mathrm{D}$ and $3 \mathrm{D}$ learning by solving a 3D task using $2 \mathrm{D}$ convolutional architectures. These methods render several views for a given 3D shape and leverage the rendered images to solve the end task. As a result, they build upon the recent advances in 2D grid-based deep learning and leverage larger image datasets for pre-training (e.g. ImageNet [67]) to compensate for the general scarcity of labeled 3D datasets. However, the manner of choosing the rendering view-points for such methods remains mostly unexplored. Current methods rely on heuristics like random sampling in scenes [45] or predefined canonical view-points in oriented datasets [75]. There is no evidence suggesting that such heuristics are empirically the best choice. To address this shortcoming, we propose to learn better view-points by introducing a MultiView Transformation Network (MVTN). As shown in Fig. 1, MVTN learns to regress view-points, renders those views with a differentiable renderer, and trains the downstream task-specific network in an end-to-end fashion, thus leading to the most suitable views for the task. MVTN is inspired 
by the Spatial Transformer Network (STN) [34], which was developed for the 2D image domain. Both MVTN and STN learn spatial transformations for the input without leveraging any extra supervision nor adjusting the learning process.

The paradigm of perception by predicting the best environment parameters that generated the image is called Vision as Inverse Graphics (VIG) [26, 44, 78, 38, 85]. One approach to VIG is to make the rendering process invertible or differentiable [57, 41, 53, 13, 51]. In this paper, MVTN takes advantage of differentiable rendering [41, 53, 66]. With such a renderer, models can be trained end-to-end for a specific target 3D vision task, with the view-points (i.e. camera poses) being inferred by MVTN in the same forward pass. To the best of our knowledge, we are the first to integrate a learnable approach to view-point prediction in multi-view methods by using a differentiable renderer and establishing an end-to-end pipeline that works for both mesh and 3D point cloud classification and retrieval.

Contributions: (i) We propose a Multi-View Transformation Network (MVTN) that regresses better view-points for multi-view methods. Our MVTN leverages a differentiable renderer that enables end-to-end training for 3D shape recognition tasks. (ii) Combining MVTN with multi-view approaches leads to state-of-the-art results in 3D classification and shape retrieval on standard benchmarks ModelNet40 [79], ShapeNet Core55 [8, 68], and ScanObjectNN [72]. (iii) Additional analysis shows that MVTN improves the robustness of multi-view approaches to rotation and occlusion, making MVTN more practical for realistic scenarios, where 3D models are not perfectly aligned or partially cropped.

\section{Related Work}

Deep Learning on 3D Data. PointNet [62] paved the way as the first deep learning algorithm to operate directly on 3D point clouds. PointNet computes point features independently and aggregates them using an order invariant function like max-pooling. Subsequent works focused on finding neighborhoods of points to define point convolutional operations [65, 74, 52, 47, 46, 73]. Voxel-based deep networks allow for 3D CNNs yet suffer from cubic memory complexity $[59,14,25]$. Several recent works combine point cloud representations with other 3D modalities like voxels [56] or multi-view images $[83,35]$. In this paper, we leverage a point encoder to predict the optimal view-points, from which images are rendered and fed to a multi-view network.

Multi-View 3D Shape Classification. The first work on using 2D images to recognize 3D objects was proposed by Bradski et al. [5]. Twenty years later and after the success of deep learning in 2D vision tasks, MVCNN [69] emerged as the first use of deep 2D CNNs for 3D object recognition. The original MVCNN uses max pooling to aggregate features from different views. Several follow-up works propose different strategies to assign weights to views to perform weighted average pooling of view-specific features [84, 82, 20, 12]. RotationNet [40] classifies the views and the object jointly. Equivariant MV-Network [18] uses a rotation equivariant convolution operation on multi-views by utilizing rotation group convolutions [15]. The more recent work of ViewGCN [75] utilizes dynamic graph convolution operations to adaptively pool features from different fixed views for the task of 3D shape classification. All these previous methods rely on fixed rendered datasets of 3D objects. The work of [12] attempts to select views adaptively through reinforcement learning and RNNs, but this comes with limited success and an elaborate training process. In this paper, we propose a novel MVTN framework for predicting optimal view-points in a multi-view setup. This is done by jointly training MVTN with a multi-view task-specific network, without the need for any extra supervision nor adjustment to the learning process.

3D Shape Retrieval. Early methods in the literature compare the distribution of hand-crafted descriptors to retrieve similar 3D shapes. Those shape signatures could either represent geometric [60] or visual [10] cues. Traditional geometric methods would estimate distributions of certain characteristics (e.g. distances, angles, areas, or volumes) to measure the similarity between shapes $[1,9,7]$. Gao et al. [22] use multiple camera projections, and Wu et al. [80] use a voxel grid to extract analogous model-based signatures. Su et al. [69] introduce a deep learning pipeline for multiview classification, with aggregated features achieving high retrieval performance. They use a low-rank Mahalanobis metric atop extracted multi-view features to improve retrieval performance. This seminal work on multi-view learning is extended for retrieval with volumetric-based descriptors [63], hierarchical view-group architectures [20], and triplet-center loss [32]. Jiang et al. [37] investigate better views for retrieval using many loops of circular cameras around the three principal axes. However, these approaches consider fixed camera view-points compared to MVTN's learnable ones.

Vision as Inverse Graphics (VIG). A key issue in VIG is the non-differentiability of the classical graphics pipeline. Recent VIG approaches focus on making the graphics operations differentiable, allowing gradients to flow from the image to the rendering parameters directly [57, 41, 53, 51, 27]. NMR [41] approximates non-differentiable rasterization by smoothing the edge rendering, where SoftRas [53] assigns a probability for all mesh triangles to every pixel in the image. Synsin [76] proposes an alpha-blending mechanism for differentiable point cloud rendering. The Pytorch3D [66] renderer improves the speed and modularity of SoftRas and Synsin and allows for customized shaders and point cloud rendering. MVTN harnesses advances in differentiable rendering to train jointly with the multi-view network in an end-to-end fashion. Using both mesh and point cloud differentiable rendering enables MVTN to work on 3D CAD models and the more accessible 3D point cloud data. 


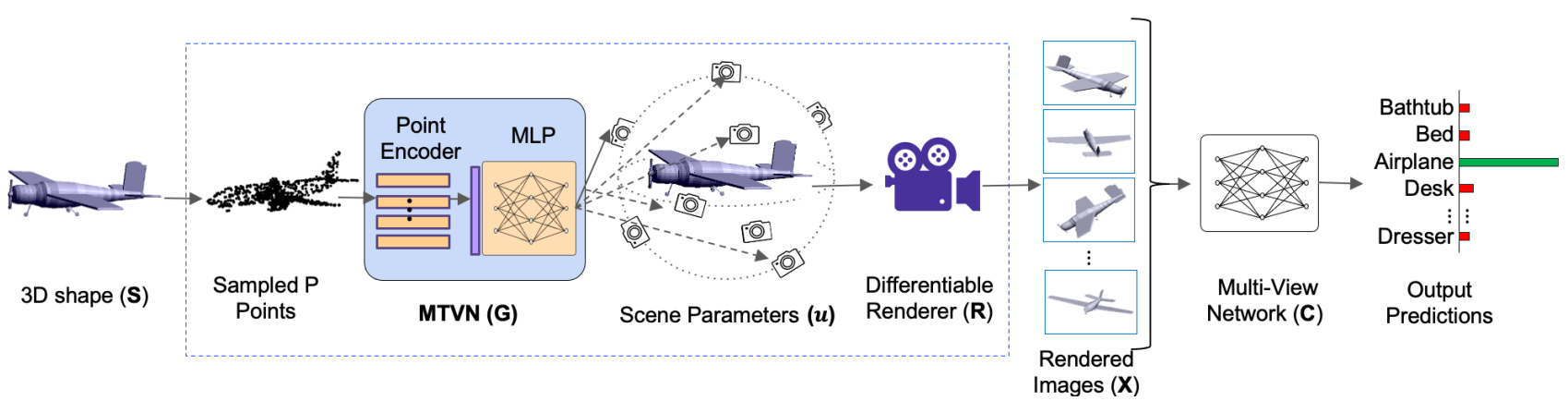

Figure 2. End-to-End Learning Pipeline for Multi-View Recognition. To learn adaptive scene parameters $\mathbf{u}$ that maximize the performance of a multi-view network $\mathbf{C}$ for every $3 \mathrm{D}$ object shape $\mathbf{S}$, we use a differentiable renderer $\mathbf{R}$. MVTN extracts coarse features from $\mathbf{S}$ by a point encoder and regresses the adaptive scene parameters for that object. In this example, the parameters $\mathbf{u}$ are the azimuth and elevation angles of cameras pointing towards the center of the object. The MVTN pipeline is optimized end-to-end for the task loss.

\section{Methodology}

We illustrate our proposed multi-view pipeline using MVTN in Fig. 2. MVTN is a generic module that learns camera view-point transformations for specific 3D multi-view tasks, e.g. 3D shape classification. In this section, we review a generic framework for common multi-view pipelines, introduce MVTN details, and present an integration of MVTN for 3D shape classification and retrieval.

\subsection{Overview of Multi-View 3D Recognition}

3D multi-view recognition defines $M$ different images $\left\{\mathbf{x}_{i}\right\}_{i=1}^{M}$ rendered from multiple view-points of the same shape $\mathbf{S}$. The views are fed into the same backbone network f that extracts discriminative features per view. These features are then aggregated among views to describe the entire shape and used for downstream tasks such as classification or retrieval. Specifically, a multi-view network $\mathbf{C}$ with parameters $\boldsymbol{\theta}_{\mathbf{C}}$ operates on an input set of images $\mathbf{X} \in \mathbb{R}^{M \times h \times w \times c}$ to obtain a softmax probability vector for the shape $\mathbf{S}$.

Training Multi-View Networks. The simplest deep multiview classifier is MVCNN, where $\mathbf{C}=\operatorname{MLP}\left(\max _{i} \mathbf{f}\left(\mathbf{x}_{i}\right)\right)$ with $\mathbf{f}: \mathbb{R}^{h \times w \times c} \rightarrow \mathbb{R}^{d}$ being a $2 \mathrm{D}$ CNN backbone (e.g. ResNet [31]) applied individually on each rendered image. A more recent method like ViewGCN would be described as $\mathbf{C}=\operatorname{MLP}\left(\operatorname{cat}_{\mathrm{GCN}}\left(\mathbf{f}\left(\mathbf{x}_{i}\right)\right)\right)$, where $\mathrm{cat}_{\mathrm{GCN}}$ is an aggregation of views' features learned from a graph convolutional network. In general, learning a task-specific multi-view network on a labeled 3D dataset is formulated as:

$$
\begin{aligned}
\underset{\boldsymbol{\theta}_{\mathbf{C}}}{\arg \min } \sum_{n}^{N} L\left(\mathbf{C}\left(\mathbf{X}_{n}\right), y_{n}\right) \\
=\underset{\boldsymbol{\theta}_{\mathbf{C}}}{\arg \min } \sum_{n}^{N} L\left(\mathbf{C}\left(\mathbf{R}\left(\mathbf{S}_{n}, \mathbf{u}_{0}\right)\right), y_{n}\right),
\end{aligned}
$$

where $L$ is a task-specific loss defined over $N$ 3D shapes in the dataset, $y_{n}$ is the label for the $n^{\text {th }} 3 \mathrm{D}$ shape $\mathbf{S}_{n}$, and $\mathbf{u}_{0} \in \mathbb{R}^{\tau}$ is a set of $\tau$ fixed scene parameters for the entire dataset. These parameters represent properties that affect the rendered image, including camera view-point, light, object color, and background. $\mathbf{R}$ is the renderer that takes as input a shape $\mathbf{S}_{n}$ and the parameters $\mathbf{u}_{0}$ to produce $M$ multi-view images $\mathbf{X}_{n}$ per shape. In our experiments, we choose the scene parameters $\mathbf{u}$ to be the azimuth and elevation angles of the camera view-points pointing towards the object center, thus setting $\tau=2 M$.

Canonical Views. Previous multi-view methods rely on scene parameters $\mathbf{u}_{0}$ that are pre-defined for the entire 3D dataset. In particular, the fixed camera view-points are usually selected based on the alignment of the 3D models in the dataset. The most common view configurations are circular that aligns view-points on a circle around the object [69, 84] and spherical that aligns equally spaced view-points on a sphere surrounding the object [75, 40]. Fixing those canonical views for all 3D objects can be misleading for some classes. For example, looking at a bed from the bottom could confuse a 3D classifier. In contrast, MVTN learns to regress per-shape view-points, as illustrated in Fig. 3.

\subsection{Multi-View Transformation Network (MVTN)}

Previous multi-view methods take the multi-view image $\mathbf{X}$ as the only representation for the $3 \mathrm{D}$ shape, where $\mathbf{X}$ is rendered using fixed scene parameters $\mathbf{u}_{0}$. In contrast, we consider a more general case, where $\mathbf{u}$ is variable yet within bounds $\pm \mathbf{u}_{\text {bound }}$. Here, $\mathbf{u}_{\text {bound }}$ is positive and it defines the permissible range for the scene parameters. We set $\mathbf{u}_{\text {bound }}$ to $180^{\circ}$ and $90^{\circ}$ for each azimuth and elevation angle.

Differentiable Renderer. A renderer $\mathbf{R}$ takes a 3D shape $\mathbf{S}$ (mesh or point cloud) and scene parameters $\mathbf{u}$ as inputs, and outputs the corresponding $M$ rendered images $\left\{\mathbf{x}_{i}\right\}_{i=1}^{M}$. Since $\mathbf{R}$ is differentiable, gradients $\frac{\partial \mathbf{x}_{i}}{\partial \mathbf{u}}$ can propagate backward from each rendered image to the scene parameters, thus establishing a framework that suits end-to-end deep learning pipelines. When $\mathbf{S}$ is represented as a $3 \mathrm{D}$ mesh, $\mathbf{R}$ has two components: a rasterizer and a shader. First, the rasterizer transforms meshes from the world to view coordinates given 


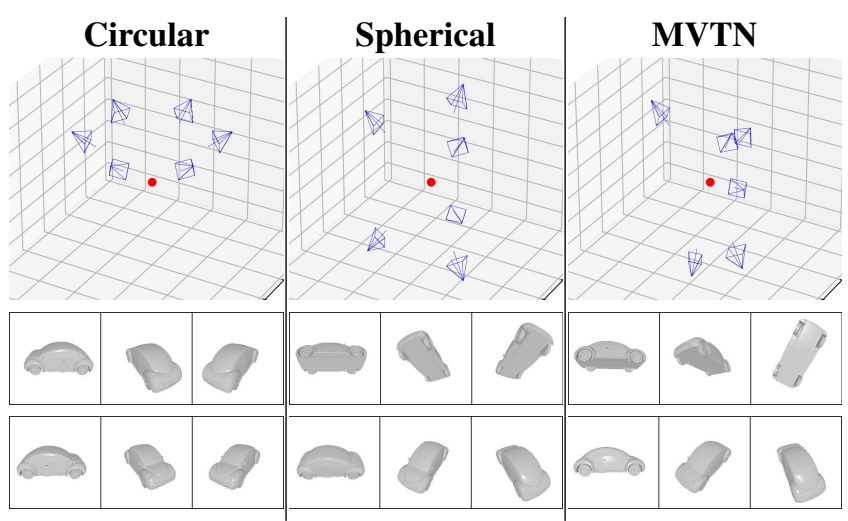

Figure 3. Multi-View Camera Configurations: The view setups commonly used in the multi-view literature are circular [69] or spherical $[75,40]$. Our MVTN learns to predict specific viewpoints for each object shape at inference time. The shape's center is shown as a red dot, and the view-points as blue cameras with their mesh renderings shown at the bottom.

the camera view-point and assigns faces to pixels. Using these face assignments, the shader creates multiple values for each pixel then blends them. On the other hand, if $\mathbf{S}$ is represented by a $3 \mathrm{D}$ point cloud, $\mathbf{R}$ would use an alpha-blending mechanism instead [76]. Fig. 3 and Fig. 4 illustrate examples of mesh and point cloud renderings used in MVTN.

View-Points Conditioned on 3D Shape. We design $\mathbf{u}$ to be a function of the 3D shape by learning a Multi-View Transformation Network (MVTN), denoted as $\mathbf{G} \in \mathbb{R}^{P \times 3} \rightarrow \mathbb{R}^{\tau}$ and parameterized by $\boldsymbol{\theta}_{\mathrm{G}}$, where $P$ is the number of points sampled from shape $\mathbf{S}$. Unlike Eq (1) that relies on constant rendering parameters, MVTN predicts $\mathbf{u}$ adaptively for each object shape $\mathbf{S}$ and is optimized along with the classifier $\mathbf{C}$. The pipeline is trained end-to-end to minimize the following loss on a dataset of $\mathrm{N}$ objects:

$$
\begin{array}{r}
\underset{\boldsymbol{\theta}_{\mathbf{C}}, \boldsymbol{\theta}_{\mathbf{G}}}{\arg \min } \sum_{n}^{N} L\left(\mathbf{C}\left(\mathbf{R}\left(\mathbf{S}_{n}, \mathbf{u}_{n}\right)\right), y_{n}\right), \\
\text { s. t. } \quad \mathbf{u}_{n}=\mathbf{u}_{\text {bound }} \cdot \tanh \left(\mathbf{G}\left(\mathbf{S}_{n}\right)\right)
\end{array}
$$

Here, $\mathbf{G}$ encodes a 3D shape to predict its optimal viewpoints for the task-specific multi-view network $\mathbf{C}$. Since the goal of $\mathbf{G}$ is only to predict view-points and not classify objects (as opposed to $\mathbf{C}$ ), its architecture is designed to be simple and light-weight. As such, we use a simple point encoder (e.g. shared MLP as in PointNet [62]) that processes $P$ points from $\mathbf{S}$ and produces coarse shape features of dimension $b$. Then, a shallow MLP regresses the scene parameters $\mathbf{u}_{n}$ from the global shape features. To force the predicted parameters $\mathbf{u}$ to be within a permissible range $\pm \mathbf{u}_{\text {bound }}$, we use a hyperbolic tangent function scaled by $\mathbf{u}_{\text {bound }}$.

MVTN for 3D Shape Classification. To train MVTN for 3D shape classification, we define a cross-entropy loss in $\mathrm{Eq}(2)$, yet other losses and regularizers can be used here as

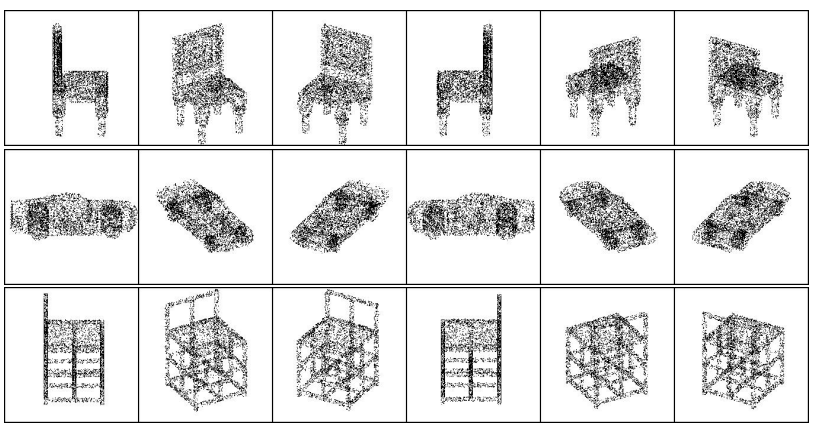

Figure 4. Multi-View Point Cloud Renderings. We show some examples of point cloud renderings used in our pipeline. Note how point cloud renderings offer more information about content hidden from the camera view-point (e.g. car wheels from the occluded side), which can be useful for recognition.

well. The multi-view network $(\mathbf{C})$ and the $\operatorname{MVTN}(\mathbf{G})$ are trained jointly on the same loss. One merit of our multi-view pipeline is its ability to seamlessly handle 3D point clouds, which is absent in previous multi-view methods. When $\mathbf{S}$ is a 3D point cloud, we simply define $\mathbf{R}$ as a differentiable point cloud renderer.

MVTN for 3D Shape Retrieval. The shape retrieval task is defined as follows: given a query shape $\mathbf{S}_{q}$, find the most similar shapes in a broader set of size $N$. For this task, we follow the retrieval setup of MVCNN [69]. In particular, we consider the deep feature representation of the last layer before the classifier in $\mathbf{C}$. We project those features into a more expressive space using LFDA reduction [70] and consider the reduced feature as the signature to describe a shape. At test time, shape signatures are used to retrieve (in order) the most similar shapes in the training set.

\section{Experiments}

We evaluate MVTN for the tasks of 3D shape classification and retrieval on ModelNet40 [79], ShapeNet Core55 [8], and the more realistic ScanObjectNN [72].

\subsection{Datasets}

ModelNet40. ModelNet40 [79] is composed of 12,311 3D objects $(9,843 / 2,468$ in training/testing) labelled with 40 object classes. Since we render 3D models in the forward pass, we limit the number of triangles in the meshes due to hardware constraints. In particular, we simplify the meshes to $20 \mathrm{k}$ vertices using the official Blender API [4, 23].

ShapeNet Core55. ShapeNet Core55 is a subset of ShapeNet [8] comprising 51,162 3D mesh objects labelled with 55 object classes. The training, validation, and test sets consist of 35764, 5133, and 10265 shapes, respectively. It is designed for the shape retrieval challenge SHREK [68].

ScanObjectNN. ScanObjectNN [72] is a recently released point cloud dataset for 3D classification that is more realistic 
and challenging than ModelNet40, since it includes background and considers occlusions. The dataset is composed of 2902 point clouds divided into 15 object categories. We consider its three main variants: object only, object with background, and the hardest perturbed variant (PB_T50_RS variant). These variants are used in the 3D Scene Understanding Benchmark associated with the ScanObjectNN dataset. This dataset offers a more challenging setup than ModelNet40 and tests the generalization capability of 3D deep learning model in more realistic scenarios.

\subsection{Metrics}

Classification Accuracy. The standard evaluation metric in 3D classification is accuracy. We report overall accuracy (percentage of correctly classified test samples) and average per-class accuracy (mean of all true class accuracies).

Retrieval mAP. Shape retrieval is evaluated by mean Average Precision (mAP) over test queries. For every query shape $\mathbf{S}_{q}$ from the test set, AP is defined as $A P=\frac{1}{\mathrm{GTP}} \sum_{n}^{N} \frac{\mathbb{1}\left(\mathbf{S}_{n}\right)}{n}$, where $G T P$ is the number of ground truth positives, $N$ is the size of the ordered training set, and $\mathbb{1}\left(\mathbf{S}_{n}\right)=1$ if the shape $\mathbf{S}_{n}$ is from the same class label of query $\mathbf{S}_{q}$. We average the retrieval AP over the test set to measure retrieval mAP.

\subsection{Baselines}

Voxel Networks. We choose VoxNet [59], DLAN [21], and 3DShapeNets [79] as baselines that use voxels.

Point Cloud Networks. We select PointNet [62], PointNet++ [65], DGCNN [74], PVNet [83], and KPConv [71] as baselines that use point clouds. These methods leverage different convolution operators on point clouds by aggregating local and global point information.

Multi-view Networks. We compare against MVCNN [69], RotationNet [40], GVCNN [20] and ViewGCN [75] as representative multi-view methods. These methods are limited to meshes, pre-rendered from canonical view-points.

\subsection{MVTN Details}

Rendering. We choose the differentiable mesh and point cloud renderers $\mathbf{R}$ from Pytorch3D [66] in our pipeline for their speed and compatibility with Pytorch libraries [61]. We show examples of the rendered images for meshes (Fig. 3) and point clouds (Fig. 4). Each rendered image has a size of $224 \times 224$. For ModelNet40, we use the differentiable mesh renderer. We direct the light randomly and assign a random color for the object for augmentation purposes in training. In testing, we keep a fixed light pointing towards the object center and color the object white for stable performance. For ShapeNet Core55 and ScanObjectNN, we use the differentiable point cloud renderer using 2048 and 5000 points, respectively. Point cloud rendering offers a light alternative to mesh rendering when the mesh contains a large number of faces that hinders training the MVTN pipeline.

\begin{tabular}{rccc}
\hline \multicolumn{1}{c}{ Method } & \multicolumn{3}{c}{ Classification Accuracy } \\
VoxNet [59] & Voxels & 83.0 & 85.9 \\
PointNet [62] & Points & 86.2 & 89.2 \\
PointNet++ [65] & Points & - & 91.9 \\
PointCNN [52] & Points & 88.1 & 91.8 \\
DGCNN [74] & Points & 90.2 & 92.2 \\
SGAS [50] & Points & - & 93.2 \\
KPConv[71] & Points & - & 92.9 \\
PTransformer[86] & Points & $\mathbf{9 0 . 6}$ & $\mathbf{9 3 . 7}$ \\
\hline MVCNN [69] & 12 Views & 90.1 & 90.1 \\
GVCNN [20] & 12 Views & 90.7 & 93.1 \\
ViewGCN [75] & 20 Views & $\mathbf{9 6 . 5}$ & $\mathbf{9 7 . 6}$ \\
\hline ViewGCN [75] $^{*}$ & 12 views & 90.7 & 93.0 \\
ViewGCN [75]* & 20 views & 91.3 & 93.3 \\
MVTN (ours) & 12 Views & 92.0 & $\mathbf{9 3 . 8}$ \\
MVTN (ours) & 20 Views & $\mathbf{9 2 . 2}$ & 93.5 \\
\hline
\end{tabular}

Table 1. 3D Shape Classification on ModelNet40. We compare MVTN against other methods in 3D classification on ModelNet40 [79]. ${ }^{*}$ indicates results from our rendering setup (differentiable pipeline), while other multi-view results are reported from prerendered views. Bold denotes the best result in its setup.

\begin{tabular}{rccc}
\hline & \multicolumn{3}{c}{ Classification Overall Accuracy } \\
Method & OBJ_BG & OBJ_ONLY & Hardest \\
\hline 3DMFV [3] & 68.2 & 73.8 & 63.0 \\
PointNet [62] & 73.3 & 79.2 & 68.0 \\
SpiderCNN [81] & 77.1 & 79.5 & 73.7 \\
PointNet ++ [65] & 82.3 & 84.3 & 77.9 \\
PointCNN [52] & 86.1 & 85.5 & 78.5 \\
DGCNN [74] & 82.8 & 86.2 & 78.1 \\
SimpleView [24] & - & - & 79.5 \\
BGA-DGCNN [72] & - & - & 79.7 \\
BGA-PN++ [72] & - & - & 80.2 \\
\hline MVTN (ours) & $\mathbf{9 2 . 6}$ & $\mathbf{9 2 . 3}$ & $\mathbf{8 2 . 8}$ \\
\hline
\end{tabular}

Table 2. 3D Point Cloud Classification on ScanObjectNN. We compare the performance of MVTN in 3D point cloud classification on three different variants of ScanObjectNN [72]. The variants include object with background, object only, and the hardest variant.

View-Point Prediction. As shown in Eq (2), the MVTN G network learns to predict the view-points directly (MVTNdirect). Alternatively, MVTN can learn relative offsets w.r.t. initial parameters $\mathbf{u}_{0}$. In this case, we concatenate the point features extracted in $\mathbf{G}$ with $\mathbf{u}_{0}$ to predict the offsets to apply on $\mathbf{u}_{0}$. The learned view-points $\mathbf{u}_{n}$ in Eq (2) are defined as: $\mathbf{u}_{n}=\mathbf{u}_{0}+\mathbf{u}_{\text {bound }} \cdot \tanh \left(\mathbf{G}\left(\mathbf{u}_{0}, \mathbf{S}_{n}\right)\right)$. We take $\mathbf{u}_{0}$ to be the circular or spherical configurations commonly used in multiview classification pipelines [69, 40, 75]. We refer to these learnable variants as MVTN-circular and MVTN-spherical, 


\begin{tabular}{rccc}
\hline \multicolumn{1}{c}{ Method } & \multicolumn{3}{c}{$\begin{array}{c}\text { Shape Retrieval (mAP) } \\
\text { Data Type }\end{array}$ ModelNet40 ShapeNet Core } \\
\hline LFD [11] & Voxels & 40.9 & - \\
3D ShapeNets [79] & Voxels & 49.2 & - \\
Densepoint[54] & Points & 88.5 & - \\
PVNet[83] & Points & 89.5 & - \\
MVCNN [69] & 12 Views & 80.2 & 73.5 \\
GIFT [2] & 20 Views & - & 64.0 \\
MVFusionNet [36] & 12 Views & - & 62.2 \\
ReVGG [68] & 20 Views & - & 74.9 \\
RotNet [40] & 20 Views & - & 77.2 \\
ViewGCN [75] & 20 Views & - & 78.4 \\
MLVCNN [37] & 24 Views & 92.2 & - \\
\hline MVTN (ours) & 12 Views & $\mathbf{9 2 . 9}$ & $\mathbf{8 2 . 9}$ \\
\hline
\end{tabular}

Table 3. 3D Shape Retrieval. We benchmark the shape retrieval mAP of MVTN on ModelNet40 [79] and ShapeNet Core55 [8, 68]. MVTN achieves the best retrieval performance among recent stateof-the-art methods on both datasets with only 12 views.

accordingly. For MVTN-circular, the initial elevations for the views are $30^{\circ}$, and the azimuth angles are equally distributed over $360^{\circ}$ following [69]. For MVTN-spherical, we follow the method from [17] that places equally-spaced viewpoints on a sphere for an arbitrary number of views, which is similar to the "dodecahedral" configuration in ViewGCN. Architecture. We select MVCNN [69], RotationNet [40], and the more recent ViewGCN [75] as our multi-view networks of choice in the MVTN pipeline. In our experiments, we select PointNet [62] as the 3D point encoder network G and experiment with DGCNN in Section 6.1. We sample $P=2048$ points from each mesh as input to the point encoder and use a 5-layer MLP for the regression network, which takes as input the point features extracted by the point encoder of size $b=40$. All MVTN variants and the baseline multi-view networks use ResNet-18 [31] pre-trained on ImageNet [67] for the multi-view backbone in $C$, with output features of size $d=1024$. The main classification and retrieval results are based on MVTN-spherical with ViewGCN [75] as the multi-view network $\mathbf{C}$, unless otherwise specified as in Section 5.3 and Section 6.1.

Training Setup. To avoid gradient instability introduced by the renderer, we use gradient clipping in the MVTN network G. We clip the gradient updates such that the $\ell_{2}$ norm of the gradients does not exceed 30 . We use a learning rate of 0.001 but refrain from fine-tuning the hyper-parameters introduced in MVCNN [69] and View-GCN [75]. More details about the training procedure are in the Appendix.

\section{Results}

The main results of MVTN are summarized in Tables 1, 2, 3 and 4. We achieve state-of-the-art performance in 3D classification on ScanObjectNN by a large margin (up to

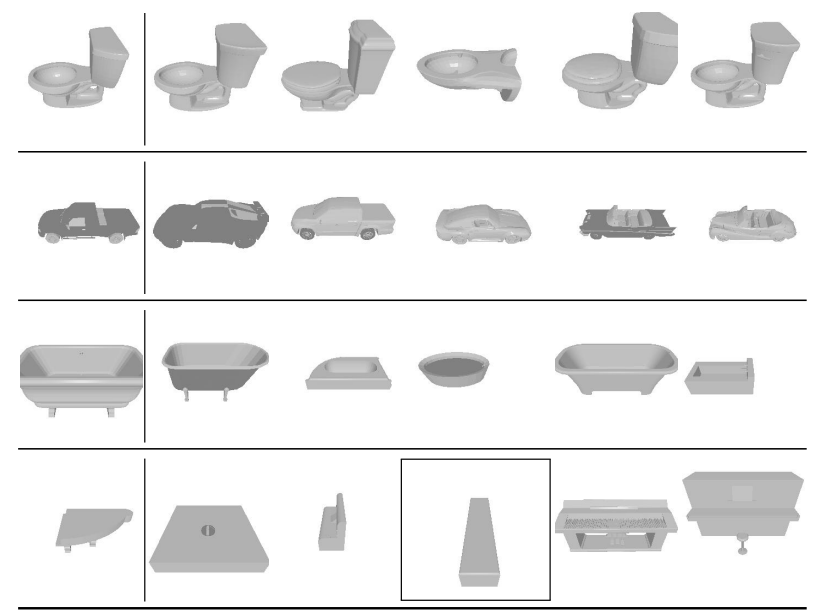

Figure 5. Qualitative Examples for Object Retrieval: (left): we show some query objects from the test set. (right): we show top five retrieved objects by our MVTN from the training set. Images of negative retrieved objects are framed.

6\%) and achieve a competitive test accuracy of $\mathbf{9 3 . 8} \%$ on ModelNet40. On shape retrieval, we achieve state-of-theart performance on both ShapeNet Core55 (82.9 mAP) and ModelNet40 (92.9 mAP). Following the common practice, we report the best results out of four runs in benchmark tables, but detailed results are in Appendix.

\subsection{D Shape Classification}

Table 1 compares the performance of MVTN against other methods on ModelNet40 [79]. Our MVTN achieves a competitive test accuracy of $93.8 \%$ compared to all previous methods. ViewGCN [75] achieves higher classification performance by relying on higher quality images from a more advanced yet non-differentiable OpenGL [77] renderer. For a fair comparison, we report with an * the performance of ViewGCN using images generated by the renderer used in MVTN. Using the same rendering process, regressing views with MVTN improves the classification performance of the baseline ViewGCN at 12 and 20 views. We believe future advances in differentiable rendering would bridge the gap between our rendered images and the original high-quality pre-rendered ones.

Table 2 reports the classification accuracy of a 12 view MVTN on the realistic ScanObjectNN benchmark [72]. MVTN improves performance on different variants of the dataset. The most difficult variant of ScanObjectNN (PB_T50_RS) includes challenging scenarios of objects undergoing translation and rotation. Our MVTN achieves stateof-the-art results $(+2.6 \%)$ on this variant, highlighting the merits of MVTN for realistic 3D point cloud scans. Also, note how adding background points (in OBJ_BG) does not hurt MVTN, contrary to most other classifiers. . 


\begin{tabular}{rccc}
\hline & \multicolumn{3}{c}{ Rotation Perturbations Range } \\
Method & $0^{\circ}$ & $\pm 90^{\circ}$ & $\pm 180^{\circ}$ \\
\hline PointNet [62] & 88.7 & 42.5 & 38.6 \\
PointNet ++ [65] & 88.2 & 47.9 & 39.7 \\
RSCNN [55] & 90.3 & 90.3 & 90.3 \\
\hline MVTN (ours) & $\mathbf{9 1 . 7}$ & $\mathbf{9 0 . 8}$ & $\mathbf{9 1 . 2}$ \\
\hline
\end{tabular}

Table 4. Rotation Robustness on ModelNet40. At test time, we randomly rotate objects in ModelNet40 around the Y-axis (gravity) with different ranges and report the overall accuracy. MVTN displays strong robustness to such Y-rotations.

\subsection{D Shape Retrieval}

Table 3 reports the retrieval mAP of MVTN compared with recent methods on ModelNet40 [79] and ShapeNet Core55 [8]. The results of the latter methods are taken from [37, 75, 83]. MVTN achieves state-of-the-art retrieval performance $(92.9 \% \mathrm{mAP})$ on ModelNet 40 . It also improves the state-of-the-art by a large margin in ShapeNet, while only using 12 views. It is important to note that the baselines in Table 3 include strong and recent methods trained specifically for retrieval, such as MLVCNN [37]. Fig. 5 shows qualitative examples of objects retrieved using MVTN.

\subsection{Rotation Robustness}

A common practice in $3 \mathrm{D}$ shape classification literature is to test the robustness of trained models to perturbations at test time. Following the same setup as [55, 28], we perturb the shapes with random rotations around the Y-axis (gravity-axis) contained within $\pm 90^{\circ}$ and $\pm 180^{\circ}$. We repeat the inference ten times for each setup and report the average performance in Table 4. The MVTN-circular variant (with MVCNN) reaches state-of-the-art performance in rotation robustness (91.2\% test accuracy) compared to more advanced methods trained in the same setup. The baseline RSCNN [55] is a strong baseline designed to be invariant to translation and rotation. In contrast, MVTN is learned in a simple setup with MVCNN without targeting rotation invariance.

\subsection{Occlusion Robustness}

To test the usefulness of MVTN in a realistic scenario, we investigate the common problem of occlusion in 3D computer vision, especially in 3D point cloud scans. Various factors lead to occlusion, including the view angle to the object, the sensor's sampling density (e.g. LiDAR), or the presence of noise in the sensor. In such realistic scenarios, deep learning models typically fail. To quantify this occlusion effect due to the viewing angle of the 3D sensor in our setup of 3D classification, we simulate realistic occlusion by cropping the object from canonical directions. We train PointNet [62], DGCNN [74], and MVTN on the

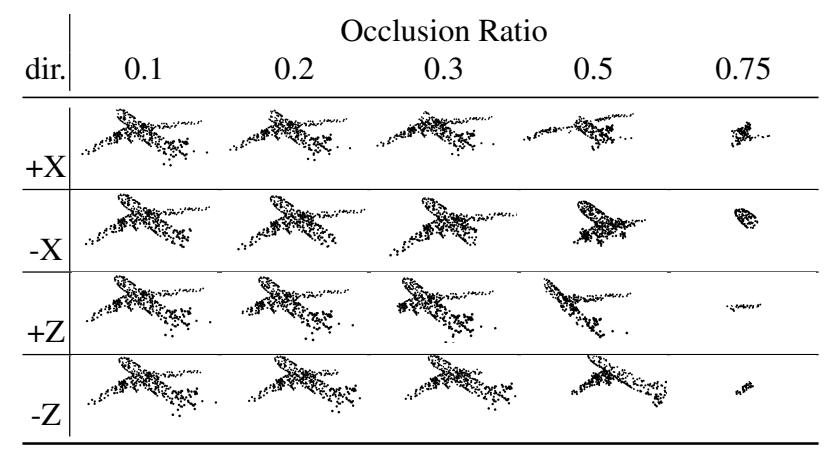

Figure 6. Occlusion of 3D Objects: We simulate realistic occlusion scenarios in $3 \mathrm{D}$ point clouds by cropping a percentage of the object along canonical directions. Here, we show an object occluded with different ratios and from different directions.

\begin{tabular}{ccccccc}
\hline \multirow{2}{*}{ Method } & 0 & 0.1 & 0.2 & 0.3 & 0.5 & 0.75 \\
\hline PointNet [62] & 89.1 & 88.2 & 86.1 & 81.6 & 53.5 & 4.7 \\
DGCNN [74] & 92.1 & 77.1 & 74.5 & 71.2 & 30.1 & 4.3 \\
\hline MVTN (ours) & $\mathbf{9 2 . 3}$ & $\mathbf{9 0 . 3}$ & $\mathbf{8 9 . 9}$ & $\mathbf{8 8 . 3}$ & $\mathbf{6 7 . 1}$ & $\mathbf{9 . 5}$ \\
\hline
\end{tabular}

Table 5. Occlusion Robustness of 3D Methods. We report the test accuracy on point cloud ModelNet40 for different occlusion ratios of the data to measure occlusion robustness of different 3D methods. MVTN achieves $13 \%$ better accuracy than PointNet (a robust network) when half of the object is occluded.

ModelNet40 point cloud dataset. Then, at test time, we crop a portion of the object (from $0 \%$ occlusion ratio to $100 \%$ ) along the $\pm \mathrm{X}, \pm \mathrm{Y}$, and $\pm \mathrm{Z}$ directions. Fig. 6 shows examples of this occlusion effect with different occlusion ratios. In all robustness experiments, the studied transformations (rotation or occlusion) happen only in test time. All the methods compared, including MVTN, are trained naturally without any augmentation by those transformations. We report the average test accuracy of the six cropping directions for the baselines and MVTN in Table 5. Note how MVTN achieves high test accuracy even when large portions of the object are cropped. Interestingly, MVTN outperforms PointNet [62] by $13 \%$ in test accuracy when half of the object is occluded. This result is significant, given that PointNet is well-known for its robustness [62, 29].

\section{Analysis and Insights}

\subsection{Ablation Study}

This section performs a comprehensive ablation study on the different components of MVTN and their effect on the overall test accuracy on ModelNet40 [79].

Number of Views. We study the effect of the number of views $M$ on the performance of MVCNN when using fixed views (circular/spherical), learned views (MVTN), and random views. The experiments are repeated four times, and the 


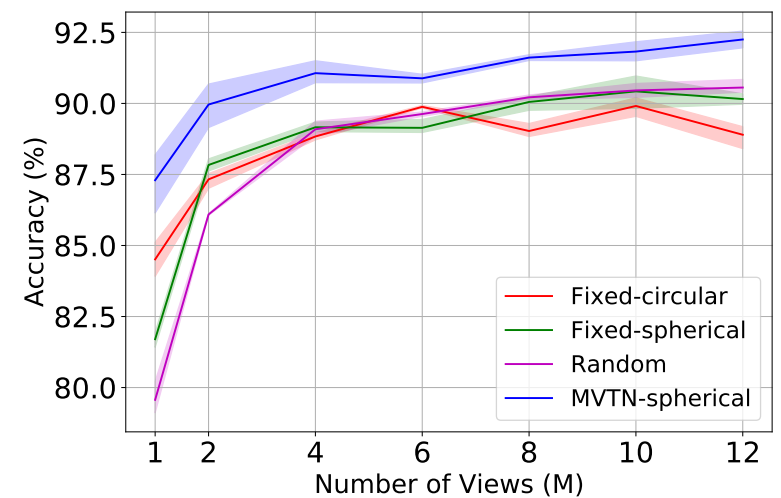

Figure 7. Effect of the Number of Views. We plot the test accuracy $v s$. the number of views (M) used to train MVCNN on fixed, random, and learned MVTN view configurations. We observe a consistent $2 \%$ improvement with MVTN over a variety of views.

\begin{tabular}{c|c|c|c}
\hline $\begin{array}{c}\text { Backbone } \\
\text { Network }\end{array}$ & $\begin{array}{c}\text { Point } \\
\text { Encoder }\end{array}$ & $\begin{array}{c}\text { MVTN } \\
\text { Setup }\end{array}$ & $\begin{array}{c}\text { Results } \\
\text { Accuracy }\end{array}$ \\
\hline \multirow{3}{*}{ ResNet-18 } & \multirow{2}{*}{ PointNet } & $\begin{array}{c}\text { circular } \\
\text { spherical }\end{array}$ & $92.83 \pm 0.06$ \\
& & $\begin{array}{c}93.41 \pm 0.13 \\
\text { circular }\end{array}$ & $93.03 \pm 0.15$ \\
& DGCNN & spherical & $93.26 \pm 0.04$ \\
\hline \multirow{3}{*}{ ResNet-34 } & \multirow{2}{*}{ PointNet } & circular & $92.72 \pm 0.16$ \\
& & spherical & $92.83 \pm 0.12$ \\
& DGCNN & circular & $92.72 \pm 0.03$ \\
& & spherical & $92.63 \pm 0.15$ \\
\hline
\end{tabular}

Table 6. Ablation Study. We analyze the effect of ablating different MVTN components on test accuracy in ModelNet40. Namely, we observe that using deeper backbone $\mathrm{CNNs}$ or a more complex point encoder do not increase the test accuracy.

average test accuracies with confidence intervals are shown in Fig. 7. The plots show how learned MVTN-spherical achieves consistently superior performance across a different number of views.

Choice of Backbone and Point Encoders. In all of our main MVTN experiments, we use ResNet-18 as our backbone and PointNet as the point feature extractor. However, different choices could be made for both. We explore using DGCNN [74] as an alternative point encoder and ResNet-34 as an alternative 2D backbone in ViewGCN. We report all MVTN ablation results in Table 6. We observe diminishing returns for making the $\mathrm{CNN}$ backbone and the shape feature extractor more complex in the MVTN setup, which justifies using the simpler combination in our main experiments

Choice of Multi-View Network. MVTN integrates smoothly with different multi-view networks and always leads to performance boost. In Table 7. we show the overall accuracies (averaged over four runs) on ModelNet40 of 12 views when fixed views are used versus when MVTN is used

\begin{tabular}{c|c|c|c}
\hline View Selection & \multicolumn{3}{|c}{ Multi-View Networks } \\
& MVCNN[69] & RotNet[40] & ViewGCN[75] \\
\hline fixed views & 90.4 & 91.6 & 93.0 \\
with MVTN & $\mathbf{9 2 . 6}$ & $\mathbf{9 3 . 2}$ & $\mathbf{9 3 . 8}$ \\
\hline
\end{tabular}

Table 7. Integrating MVTN with Multi-View Networks. We show overall classification accuracies on ModelNet40 with 12 views on different multi-view networks when fixed views are used versus when MVTN is used.

\begin{tabular}{r|ccc}
\hline \multicolumn{1}{c}{ Network } & GFLOPs & Time $(\mathrm{ms})$ & Parameters \# (M) \\
\hline MVCNN [69] & 43.72 & 39.89 & 11.20 \\
ViewGCN [75] & 44.19 & 26.06 & 23.56 \\
\hline MVTN module & $\mathbf{1 . 7 8}$ & $\mathbf{4 . 2 4}$ & $\mathbf{3 . 5}$ \\
\hline
\end{tabular}

Table 8. Time and Memory Requirements. We assess the contribution of the MVTN module to the time and memory requirements in the multi-view pipeline. We note that the MVTN's time and memory requirements are negligible.

with different multi-view networks.

Other Factors Affecting MVTN. We study the effect of the light direction in the renderer, the camera's distance to the object, and the object's color. We also study the transferability of the learned views from one multi-view network to another, and the performance of MVTN variants. More details are provided in the Appendix.

\subsection{Time and Memory Requirements}

We compare the time and memory requirements of different parts of our 3D recognition pipeline. We record the number of floating-point operations (GFLOPs) and the time of a forward pass for a single input sample. In Table 8, MVTN contributes negligibly to the time and memory requirements of the multi-view networks.

\section{Conclusions and Future Work}

Current multi-view methods rely on fixed views aligned with the dataset. We propose MVTN that learns to regress view-points for any multi-view network in a fully differentiable pipeline. MVTN harnesses recent developments in differentiable rendering and does not require any extra training supervision. Empirical results highlight the benefits of MVTN in 3D classification and 3D shape retrieval. Some possible future works for MVTN include extending it to other 3D vision tasks such as shape and scene segmentation. Furthermore, MVTN can include more intricate scene parameters different from the camera view-points, such as light and textures.

Acknowledgments. This work was supported by the King Abdullah University of Science and Technology (KAUST) Office of Sponsored Research through the Visual Computing Center (VCC) funding. 


\section{References}

[1] Ceyhun Burak Akgül, Bülent Sankur, Yücel Yemez, and Francis Schmitt. 3d model retrieval using probability densitybased shape descriptors. IEEE transactions on pattern analysis and machine intelligence, 31(6):1117-1133, 2009. 2

[2] Song Bai, Xiang Bai, Zhichao Zhou, Zhaoxiang Zhang, and Longin Jan Latecki. Gift: A real-time and scalable 3d shape search engine. In Proceedings of the IEEE conference on computer vision and pattern recognition, pages 5023-5032, 2016. 6, 20

[3] Yizhak Ben-Shabat, Michael Lindenbaum, and Anath Fischer. 3dmfv: Three-dimensional point cloud classification in realtime using convolutional neural networks. IEEE Robotics and Automation Letters, 3(4):3145-3152, 2018. 5, 19

[4] Blender Online Community. Blender - a 3D modelling and rendering package. Blender Foundation, Blender Institute, Amsterdam, 2018. 4

[5] Gary Bradski and Stephen Grossberg. Recognition of 3-d objects from multiple 2-d views by a self-organizing neural architecture. In From Statistics to Neural Networks, pages 349-375. Springer, 1994. 2

[6] Andrew Brock, Theodore Lim, James M Ritchie, and Nick Weston. Generative and discriminative voxel modeling with convolutional neural networks. arXiv preprint arXiv:1608.04236, 2016. 19

[7] Alexander M Bronstein, Michael M Bronstein, Leonidas J Guibas, and Maks Ovsjanikov. Shape google: Geometric words and expressions for invariant shape retrieval. ACM Transactions on Graphics (TOG), 30(1):1-20, 2011. 2

[8] Angel X. Chang, Thomas Funkhouser, Leonidas Guibas, Pat Hanrahan, Qixing Huang, Zimo Li, Silvio Savarese, Manolis Savva, Shuran Song, Hao Su, Jianxiong Xiao, Li Yi, and Fisher Yu. ShapeNet: An Information-Rich 3D Model Repository. Technical Report arXiv:1512.03012 [cs.GR], Stanford University - Princeton University - Toyota Technological Institute at Chicago, 2015. 2, 4, 6, 7, 13, 15, 18, 20

[9] Siddhartha Chaudhuri and Vladlen Koltun. Data-driven suggestions for creativity support in $3 \mathrm{~d}$ modeling. In ACM SIGGRAPH Asia 2010 papers, pages 1-10. 2010. 2

[10] Ding-Yun Chen, Xiao-Pei Tian, Yu-Te Shen, and Ming Ouhyoung. On visual similarity based $3 \mathrm{~d}$ model retrieval. In Computer graphics forum, volume 22, pages 223-232. Wiley Online Library, 2003. 2

[11] Ding-Yun Chen, Xiao-Pei Tian, Yu-Te Shen, and Ming Ouhyoung. On visual similarity based $3 \mathrm{~d}$ model retrieval. In Computer graphics forum, volume 22, pages 223-232. Wiley Online Library, 2003. 6, 19, 20

[12] Songle Chen, Lintao Zheng, Yan Zhang, Zhixin Sun, and Kai $\mathrm{Xu}$. Veram: View-enhanced recurrent attention model for 3d shape classification. IEEE transactions on visualization and computer graphics, 25(12):3244-3257, 2018. 2

[13] Wenzheng Chen, Huan Ling, Jun Gao, Edward Smith, Jaakko Lehtinen, Alec Jacobson, and Sanja Fidler. Learning to predict $3 \mathrm{~d}$ objects with an interpolation-based differentiable renderer. In Advances in Neural Information Processing Systems, pages 9609-9619, 2019. 2
[14] Christopher Choy, JunYoung Gwak, and Silvio Savarese. 4d spatio-temporal convnets: Minkowski convolutional neural networks. In Proceedings of the IEEE Conference on Computer Vision and Pattern Recognition, pages 3075-3084, 2019. 1,2

[15] Taco Cohen and Max Welling. Group equivariant convolutional networks. In International conference on machine learning, pages 2990-2999, 2016. 2

[16] Angela Dai and Matthias Nießner. 3dmv: Joint 3d-multi-view prediction for $3 \mathrm{~d}$ semantic scene segmentation. In Proceedings of the European Conference on Computer Vision (ECCV), pages $452-468,2018.1$

[17] Markus Deserno. How to generate equidistributed points on the surface of a sphere. If Polymerforshung (Ed.), page 99, 2004. 6

[18] Carlos Esteves, Yinshuang Xu, Christine Allen-Blanchette, and Kostas Daniilidis. Equivariant multi-view networks. In Proceedings of the IEEE International Conference on Computer Vision, pages 1568-1577, 2019. 2

[19] Yutong Feng, Yifan Feng, Haoxuan You, Xibin Zhao, and Yue Gao. Meshnet: Mesh neural network for 3d shape representation. In Proceedings of the AAAI Conference on Artificial Intelligence, volume 33, pages 8279-8286, 2019. 1

[20] Yifan Feng, Zizhao Zhang, Xibin Zhao, Rongrong Ji, and Yue Gao. Gvcnn: Group-view convolutional neural networks for $3 \mathrm{~d}$ shape recognition. In Proceedings of the IEEE Conference on Computer Vision and Pattern Recognition, pages 264-272, 2018. 2, 5, 19

[21] Takahiko Furuya and Ryutarou Ohbuchi. Deep aggregation of local 3d geometric features for $3 \mathrm{~d}$ model retrieval. In $B M V C$, volume 7, page 8, 2016. 5, 20

[22] Yue Gao, Jinhui Tang, Richang Hong, Shuicheng Yan, Qionghai Dai, Naiyao Zhang, and Tat-Seng Chua. Camera constraint-free view-based 3-d object retrieval. IEEE Transactions on Image Processing, 21(4):2269-2281, 2011. 2

[23] Michael Garland and Paul S Heckbert. Surface simplification using quadric error metrics. In Proceedings of the 24th annual conference on Computer graphics and interactive techniques, pages 209-216, 1997. 4

[24] Ankit Goyal, Hei Law, Bowei Liu, Alejandro Newell, and Jia Deng. Revisiting point cloud shape classification with a simple and effective baseline. In ICML, 2021. 5, 19

[25] Benjamin Graham, Martin Engelcke, and Laurens Van Der Maaten. 3d semantic segmentation with submanifold sparse convolutional networks. In Proceedings of the IEEE conference on computer vision and pattern recognition, pages 9224-9232, 2018. 1, 2

[26] Ulf Grenander. Pattern analysis: lectures in pattern theory, volume I. Springer, 1978. 2

[27] Abdullah Hamdi and Bernard Ghanem. Towards analyzing semantic robustness of deep neural networks. In European Conference on Computer Vision, pages 22-38. Springer, 2020. 2

[28] Abdullah Hamdi, Matthias Muller, and Bernard Ghanem. SADA: semantic adversarial diagnostic attacks for autonomous applications. In AAAI Conference on Artificial Intelligence, 2020. 7 
[29] Abdullah Hamdi, Sara Rojas, Ali Thabet, and Bernard Ghanem. Advpc: Transferable adversarial perturbations on $3 \mathrm{~d}$ point clouds. In Computer Vision - ECCV 2020, pages 241-257, Cham, 2020. Springer International Publishing. 7, 18

[30] Rana Hanocka, Amir Hertz, Noa Fish, Raja Giryes, Shachar Fleishman, and Daniel Cohen-Or. Meshenn: a network with an edge. ACM Transactions on Graphics (TOG), 38(4):1-12, 2019. 1

[31] Kaiming He, Xiangyu Zhang, Shaoqing Ren, and Jian Sun. Deep residual learning for image recognition. CoRR, abs/1512.03385, 2015. 3, 6, 13, 24

[32] Xinwei He, Yang Zhou, Zhichao Zhou, Song Bai, and Xiang Bai. Triplet-center loss for multi-view $3 \mathrm{~d}$ object retrieval. In Proceedings of the IEEE Conference on Computer Vision and Pattern Recognition, pages 1945-1954, 2018. 2

[33] Vishakh Hegde and Reza Zadeh. Fusionnet: 3d object classification using multiple data representations. arXiv preprint arXiv:1607.05695, 2016. 19

[34] Max Jaderberg, Karen Simonyan, Andrew Zisserman, et al. Spatial transformer networks. In Advances in neural information processing systems, pages 2017-2025, 2015. 2

[35] Maximilian Jaritz, Jiayuan Gu, and Hao Su. Multi-view pointnet for $3 \mathrm{~d}$ scene understanding. In Proceedings of the IEEE International Conference on Computer Vision Workshops, pages $0-0,2019.2$

[36] Kui Jia, Jiehong Lin, Mingkui Tan, and Dacheng Tao. Deep multi-view learning using neuron-wise correlationmaximizing regularizers. IEEE Transactions on Image Processing, 28(10):5121-5134, 2019. 6, 20

[37] Jianwen Jiang, Di Bao, Ziqiang Chen, Xibin Zhao, and Yue Gao. Mlvcnn: Multi-loop-view convolutional neural network for $3 \mathrm{~d}$ shape retrieval. In Proceedings of the AAAI Conference on Artificial Intelligence, volume 33, pages 8513-8520, 2019. 2, 6, 7, 20

[38] Danilo Jimenez Rezende, S. M. Ali Eslami, Shakir Mohamed, Peter Battaglia, Max Jaderberg, and Nicolas Heess. Unsupervised learning of $3 \mathrm{~d}$ structure from images. In D. D. Lee, M. Sugiyama, U. V. Luxburg, I. Guyon, and R. Garnett, editors, Advances in Neural Information Processing Systems 29, pages 4996-5004. Curran Associates, Inc., 2016. 2

[39] Evangelos Kalogerakis, Melinos Averkiou, Subhransu Maji, and Siddhartha Chaudhuri. 3d shape segmentation with projective convolutional networks. In proceedings of the IEEE conference on computer vision and pattern recognition, pages 3779-3788, 2017. 1

[40] Asako Kanezaki, Yasuyuki Matsushita, and Yoshifumi Nishida. Rotationnet: Joint object categorization and pose estimation using multiviews from unsupervised viewpoints. In Proceedings of the IEEE Conference on Computer Vision and Pattern Recognition, pages 5010-5019, 2018. 1, 2, 3, 4, 5, 6, 8, 20, 26, 27

[41] Hiroharu Kato, Yoshitaka Ushiku, and Tatsuya Harada. Neural 3d mesh renderer. In Proceedings of the IEEE Conference on Computer Vision and Pattern Recognition (CVPR), pages 3907-3916, 2018. 2
[42] Michael Kazhdan, Thomas Funkhouser, and Szymon Rusinkiewicz. Rotation invariant spherical harmonic representation of $3 \mathrm{~d}$ shape descriptors. In Symposium on geometry processing, volume 6, pages 156-164, 2003. 19, 20

[43] Roman Klokov and Victor Lempitsky. Escape from cells: Deep kd-networks for the recognition of $3 \mathrm{~d}$ point cloud models. In Proceedings of the IEEE International Conference on Computer Vision, pages 863-872, 2017. 19

[44] Tejas D Kulkarni, William F Whitney, Pushmeet Kohli, and Josh Tenenbaum. Deep convolutional inverse graphics network. In Advances in neural information processing systems (NIPS), pages 2539-2547, 2015. 2

[45] Abhijit Kundu, Xiaoqi Yin, Alireza Fathi, David Ross, Brian Brewington, Thomas Funkhouser, and Caroline Pantofaru. Virtual multi-view fusion for $3 \mathrm{~d}$ semantic segmentation. arXiv preprint arXiv:2007.13138, 2020. 1

[46] Loic Landrieu and Mohamed Boussaha. Point cloud oversegmentation with graph-structured deep metric learning. pages 7440-7449, 2019. 2

[47] Loic Landrieu and Martin Simonovsky. Large-scale point cloud semantic segmentation with superpoint graphs. In Proceedings of the IEEE Conference on Computer Vision and Pattern Recognition (CVPR), pages 4558-4567, 2018. 2

[48] Alex Lenail. Nn-svg, 2020. 17

[49] Bo Li and Henry Johan. 3d model retrieval using hybrid features and class information. Multimedia tools and applications, 62(3):821-846, 2013. 20

[50] Guohao Li, Guocheng Qian, Itzel C Delgadillo, Matthias Muller, Ali Thabet, and Bernard Ghanem. Sgas: Sequential greedy architecture search. In Proceedings of the IEEE/CVF Conference on Computer Vision and Pattern Recognition, pages 1620-1630, 2020. 5

[51] Tzu-Mao Li, Miika Aittala, Frédo Durand, and Jaakko Lehtinen. Differentiable monte carlo ray tracing through edge sampling. In SIGGRAPH Asia 2018 Technical Papers, page 222. ACM, 2018. 2

[52] Yangyan Li, Rui Bu, Mingchao Sun, Wei Wu, Xinhan Di, and Baoquan Chen. Pointenn: Convolution on X-transformed points. In Advances in neural information processing systems (NIPS), pages 820-830, 2018. 2, 5, 19

[53] Shichen Liu, Tianye Li, Weikai Chen, and Hao Li. Soft rasterizer: A differentiable renderer for image-based $3 \mathrm{~d}$ reasoning. In Proceedings of the IEEE International Conference on Computer Vision, pages 7708-7717, 2019. 2

[54] Yongcheng Liu, Bin Fan, Gaofeng Meng, Jiwen Lu, Shiming Xiang, and Chunhong Pan. Densepoint: Learning densely contextual representation for efficient point cloud processing. In Proceedings of the IEEE/CVF International Conference on Computer Vision, pages 5239-5248, 2019. 6

[55] Yongcheng Liu, Bin Fan, Shiming Xiang, and Chunhong Pan. Relation-shape convolutional neural network for point cloud analysis. In Proceedings of the IEEE Conference on Computer Vision and Pattern Recognition, pages 8895-8904, 2019. 7, 18

[56] Zhijian Liu, Haotian Tang, Yujun Lin, and Song Han. Pointvoxel cnn for efficient 3d deep learning. In Advances in Neural Information Processing Systems, pages 965-975, 2019. 2 
[57] Matthew M Loper and Michael J Black. Opendr: An approximate differentiable renderer. In European Conference on Computer Vision (ECCV), pages 154-169. Springer, 2014. 2

[58] Ilya Loshchilov and Frank Hutter. Decoupled weight decay regularization. arXiv preprint arXiv:1711.05101, 2017. 13

[59] Daniel Maturana and Sebastian Scherer. Voxnet: A 3d convolutional neural network for real-time object recognition. In 2015 IEEE/RSJ International Conference on Intelligent Robots and Systems (IROS), pages 922-928. IEEE, 2015. 1, 2, 5,19

[60] Robert Osada, Thomas Funkhouser, Bernard Chazelle, and David Dobkin. Shape distributions. ACM Transactions on Graphics (TOG), 21(4):807-832, 2002. 2

[61] Adam Paszke, Sam Gross, Soumith Chintala, Gregory Chanan, Edward Yang, Zachary DeVito, Zeming Lin, Alban Desmaison, Luca Antiga, and Adam Lerer. Automatic differentiation in pytorch. In NIPS-W, 2017. 5

[62] Charles R Qi, Hao Su, Kaichun Mo, and Leonidas J Guibas. Pointnet: Deep learning on point sets for 3d classification and segmentation. In Proceedings of the IEEE Conference on Computer Vision and Pattern Recognition (CVPR), pages 652-660, 2017. 1, 2, 4, 5, 6, 7, 13, 18, 19, 22, 24

[63] Charles R Qi, Hao Su, Matthias Nießner, Angela Dai, Mengyuan Yan, and Leonidas J Guibas. Volumetric and multi-view cnns for object classification on 3d data. In Proceedings of the IEEE conference on computer vision and pattern recognition, pages 5648-5656, 2016. 2

[64] Charles R Qi, Hao Su, Matthias Nießner, Angela Dai, Mengyuan Yan, and Leonidas J Guibas. Volumetric and multi-view cnns for object classification on 3d data. In Proceedings of the IEEE conference on computer vision and pattern recognition, pages 5648-5656, 2016. 19

[65] Charles Ruizhongtai Qi, Li Yi, Hao Su, and Leonidas J Guibas. Pointnet++: Deep hierarchical feature learning on point sets in a metric space. In Advances in neural information processing systems (NIPS), pages 5099-5108, 2017. 1, $2,5,7,19$

[66] Nikhila Ravi, Jeremy Reizenstein, David Novotny, Taylor Gordon, Wan-Yen Lo, Justin Johnson, and Georgia Gkioxari. Accelerating 3d deep learning with pytorch3d. arXiv:2007.08501, 2020. 2, 5, 18

[67] Olga Russakovsky, Jia Deng, Hao Su, Jonathan Krause, Sanjeev Satheesh, Sean Ma, Zhiheng Huang, Andrej Karpathy, Aditya Khosla, Michael S. Bernstein, Alexander C. Berg, and Fei-Fei Li. Imagenet large scale visual recognition challenge. CoRR, abs/1409.0575, 2014. 1, 6

[68] Konstantinos Sfikas, Theoharis Theoharis, and Ioannis Pratikakis. Exploiting the PANORAMA Representation for Convolutional Neural Network Classification and Retrieval. In Ioannis Pratikakis, Florent Dupont, and Maks Ovsjanikov, editors, Eurographics Workshop on 3D Object Retrieval, pages 1-7. The Eurographics Association, 2017. 2, 4, 6, $13,18,20$

[69] Hang Su, Subhransu Maji, Evangelos Kalogerakis, and Erik Learned-Miller. Multi-view convolutional neural networks for $3 \mathrm{~d}$ shape recognition. In Proceedings of the IEEE international conference on computer vision, pages 945-953, 2015. $1,2,3,4,5,6,8,13,18,19,20,22,24,26,27$
[70] Masashi Sugiyama. Dimensionality reduction of multimodal labeled data by local fisher discriminant analysis. Journal of machine learning research, 8(May):1027-1061, 2007. 4

[71] Hugues Thomas, Charles R Qi, Jean-Emmanuel Deschaud, Beatriz Marcotegui, François Goulette, and Leonidas J Guibas. Kpconv: Flexible and deformable convolution for point clouds. In Proceedings of the IEEE/CVF International Conference on Computer Vision, pages 6411-6420, 2019. 5, 19

[72] Mikaela Angelina Uy, Quang-Hieu Pham, Binh-Son Hua, Duc Thanh Nguyen, and Sai-Kit Yeung. Revisiting point cloud classification: A new benchmark dataset and classification model on real-world data. In International Conference on Computer Vision (ICCV), 2019. 2, 4, 5, 6, 13, 16, 18, 19

[73] Weiyue Wang, Ronald Yu, Qiangui Huang, and Ulrich Neumann. Sgpn: Similarity group proposal network for 3d point cloud instance segmentation. In Proceedings of the IEEE Conference on Computer Vision and Pattern Recognition (CVPR), pages 2569-2578, 2018. 2

[74] Yue Wang, Yongbin Sun, Ziwei Liu, Sanjay E. Sarma, Michael M. Bronstein, and Justin M. Solomon. Dynamic graph cnn for learning on point clouds. ACM Transactions on Graphics (TOG), 2019. 1, 2, 5, 7, 8, 18, 19, 24

[75] Xin Wei, Ruixuan Yu, and Jian Sun. View-gen: View-based graph convolutional network for $3 \mathrm{~d}$ shape analysis. In Proceedings of the IEEE/CVF Conference on Computer Vision and Pattern Recognition, pages 1850-1859, 2020. 1, 2, 3, 4, 5, 6, 7, 8, 13, 18, 19, 20, 26, 27

[76] Olivia Wiles, Georgia Gkioxari, Richard Szeliski, and Justin Johnson. Synsin: End-to-end view synthesis from a single image. In Proceedings of the IEEE/CVF Conference on Computer Vision and Pattern Recognition, pages 7467-7477, 2020. 2,4

[77] Mason Woo, Jackie Neider, Tom Davis, and Dave Shreiner. OpenGL Programming Guide: The Official Guide to Learning OpenGL, Release 1. Addison-wesley, 1998. 6, 18

[78] Jiajun Wu, Joshua B Tenenbaum, and Pushmeet Kohli. Neural Scene De-rendering. In IEEE Conference on Computer Vision and Pattern Recognition (CVPR), 2017. 2

[79] Zhirong Wu, S. Song, A. Khosla, Fisher Yu, Linguang Zhang, Xiaoou Tang, and J. Xiao. 3d shapenets: A deep representation for volumetric shapes. In 2015 IEEE Conference on Computer Vision and Pattern Recognition (CVPR), pages 1912-1920, 2015. 2, 4, 5, 6, 7, 18, 19, 20, 22

[80] Zhirong Wu, Shuran Song, Aditya Khosla, Fisher Yu, Linguang Zhang, Xiaoou Tang, and Jianxiong Xiao. 3d shapenets: A deep representation for volumetric shapes. In Proceedings of the IEEE conference on computer vision and pattern recognition, pages 1912-1920, 2015. 2

[81] Yifan Xu, Tianqi Fan, Mingye Xu, Long Zeng, and Yu Qiao. Spidercnn: Deep learning on point sets with parameterized convolutional filters. In Proceedings of the European Conference on Computer Vision (ECCV), pages 87-102, 2018. 5, 19

[82] Ze Yang and Liwei Wang. Learning relationships for multiview 3d object recognition. In Proceedings of the IEEE International Conference on Computer Vision, pages 75057514, 2019. 2 
[83] Haoxuan You, Yifan Feng, Rongrong Ji, and Yue Gao. Pvnet: A joint convolutional network of point cloud and multi-view for $3 \mathrm{~d}$ shape recognition. In Proceedings of the 26th ACM international conference on Multimedia, pages 1310-1318, 2018. 2, 5, 6, 7, 19, 20

[84] Tan Yu, Jingjing Meng, and Junsong Yuan. Multi-view harmonized bilinear network for $3 \mathrm{~d}$ object recognition. In Proceedings of the IEEE Conference on Computer Vision and Pattern Recognition, pages 186-194, 2018. 2, 3

[85] Ye Yu and William AP Smith. Inverserendernet: Learning single image inverse rendering. arXiv preprint arXiv:1811.12328, 2018. 2

[86] Hengshuang Zhao, Li Jiang, Jiaya Jia, Philip Torr, and Vladlen Koltun. Point transformer. arXiv preprint arXiv:2012.09164, 2020. 5, 19 


\section{A. Detailed Experimental Setup}

\section{A.1. Datasets}

ModelNet40. We show in Fig. 8 examples of the mesh renderings of ModelNet40 used in training our MVTN. Note that the color of the object and the light direction are randomized in training for augmentation but are fixed in testing for stable performance.

ShapeNet Core55. In Fig. 9, we show examples of the point cloud renderings of ShapeNet Core55 [8, 68] used in training MVTN. Note how point cloud renderings offer more information about content hidden from the camera view-point, which can be useful for recognition. White color is used in training and testing for all point cloud renderings. For visualization purposes, colors are inverted in the main paper examples (Fig. 4 in the main paper).

ScanObjectNN. ScanObjectNN [72] has three main variants: object only, object with background, and the PB_T50_RS variant (hardest perturbed variant). Fig. 10 show examples of multi-view renderings of different samples of the dataset from its three variants. Note that adding the background points to the rendering gives some clues to our MVTN about the object, which explains why adding background improves the performance of MVTN in Table 10.

\section{A.2. MVTN Details}

MVTN Rendering. Point cloud rendering offers a light alternative to mesh rendering in ShapeNet because its meshes contain large numbers of faces that hinders training the MVTN pipeline. Simplifying theses "high-poly" meshes (similar to ModelNet40) results in corrupted shapes that lose their main visual clues. Therefore, we use point cloud rendering for ShapeNet, allowing to process all shapes with equal memory requirements. Another benefit of point cloud rendering is making it possible to train MVTN with a large batch size on the same GPU (bath size of 30 on V100 GPU). MVTN Architecture. We incorporate our MVTN into MVCNN [69] and ViewGCN [75]. In our experiments, we select PointNet [62] as the default point encoder of MVTN. All MVTNs and their baseline multi-view networks use ResNet18 [31] as backbone in our main experiments with output feature size $d=1024$. The azimuth angle maximum range $\left(\mathbf{u}_{\text {bound }}\right.$ ) is $\frac{180^{\circ}}{M}$ for MVTN-circular and MVTNspherical, while it is $180^{\circ}$ for MVTN-direct. On the other hand, the elevation angle maximum range ( $\mathbf{u}_{\text {bound }}$ ) is $90^{\circ}$. We use a 4-layer MLP for MVTN's regression network G. For MVTN-spherical/MVTN-spherical, the regression network takes as input $M$ azimuth angles, $M$ elevation angles, and the point features of shape $\mathbf{S}$ of size $b=40$. The widths of the MVTN networks are illustrated in Fig. 11.MVTN concatenates all of its inputs, and the MLP outputs the offsets to the initial $2 \times M$ azimuth and elevation angles. The size of the MVTN network (with $b=40$ ) is $14 M^{2}+211 M+3320$ parameters, where $M$ is the number of views. It is a shallow network of only around $9 \mathrm{~K}$ parameters when $M=12$.

View-Points. In Fig. 12, we show the basic views configurations for $M$ views previously used in the literature: circular, spherical, and random. MVTN's learned views are shown later in C.1 Since ViewGCN uses view sampling as a core operation, it requires the number of views to be at least 12, and hence, our MVTN with ViewGCN follows accordingly. Training MVTN. We use AdamW [58] for our MVTN networks with a learning rate of 0.001 . For other training details (e.g. training epochs and optimization), we follow the previous works [75, 69] for a fair comparison. The training of MVTN with MVCNN is done in 100 epochs and a batch size of 20, while the MVTN with ViewGCN is performed in two stages as proposed in the official code of the paper [75]. The first stage is 50 epochs of training the backbone CNN on the single view images, while the second stage is 35 epochs on the multi-view network on the $M$ views of the 3D object. We use learning rates of 0.0003 for MVCNN and 0.001 for ViewGCN, and a ResNet-18 [31] as the backbone CNN for both baselines and our MVTN-based networks. A weight decay of 0.01 is applied for both the multi-view network and in the MVTN networks. Due to gradient instability from the renderer, we introduce gradient clipping in the MVTN to limit the $\ell_{2}$ norm of gradient updates to 30 forG. The code is available at https://github.com/ajhamdi/MVTN. 


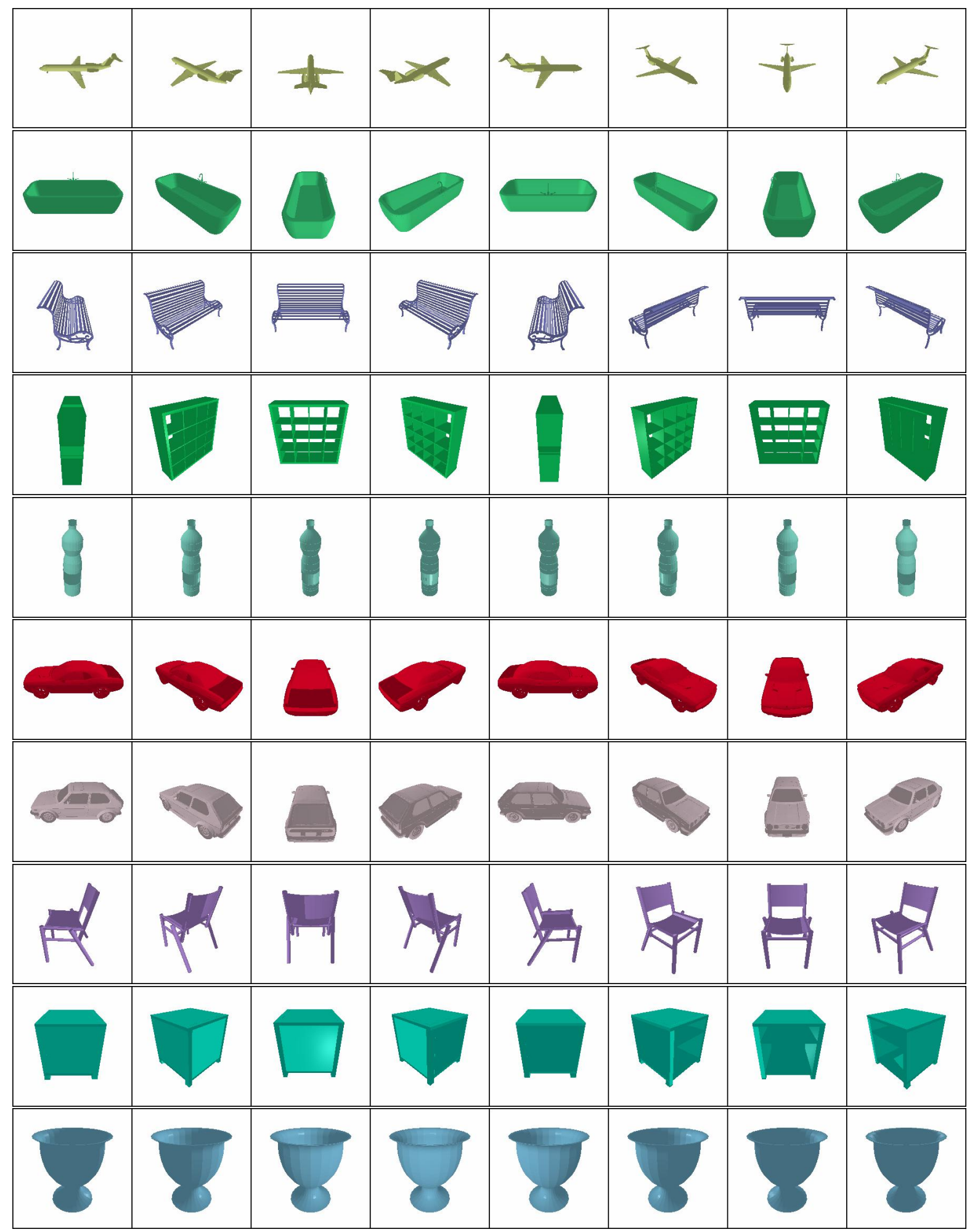

Figure 8. Training Data with Randomized Color and Lighting. We show examples of mesh renderings of ModelNet 40 used in training our MVTN. The color of the object and the light's direction are randomized during training for augmentation purposes and fixed in testing for stable performance. For this figure, eight circular views are shown for each 3D shape. 


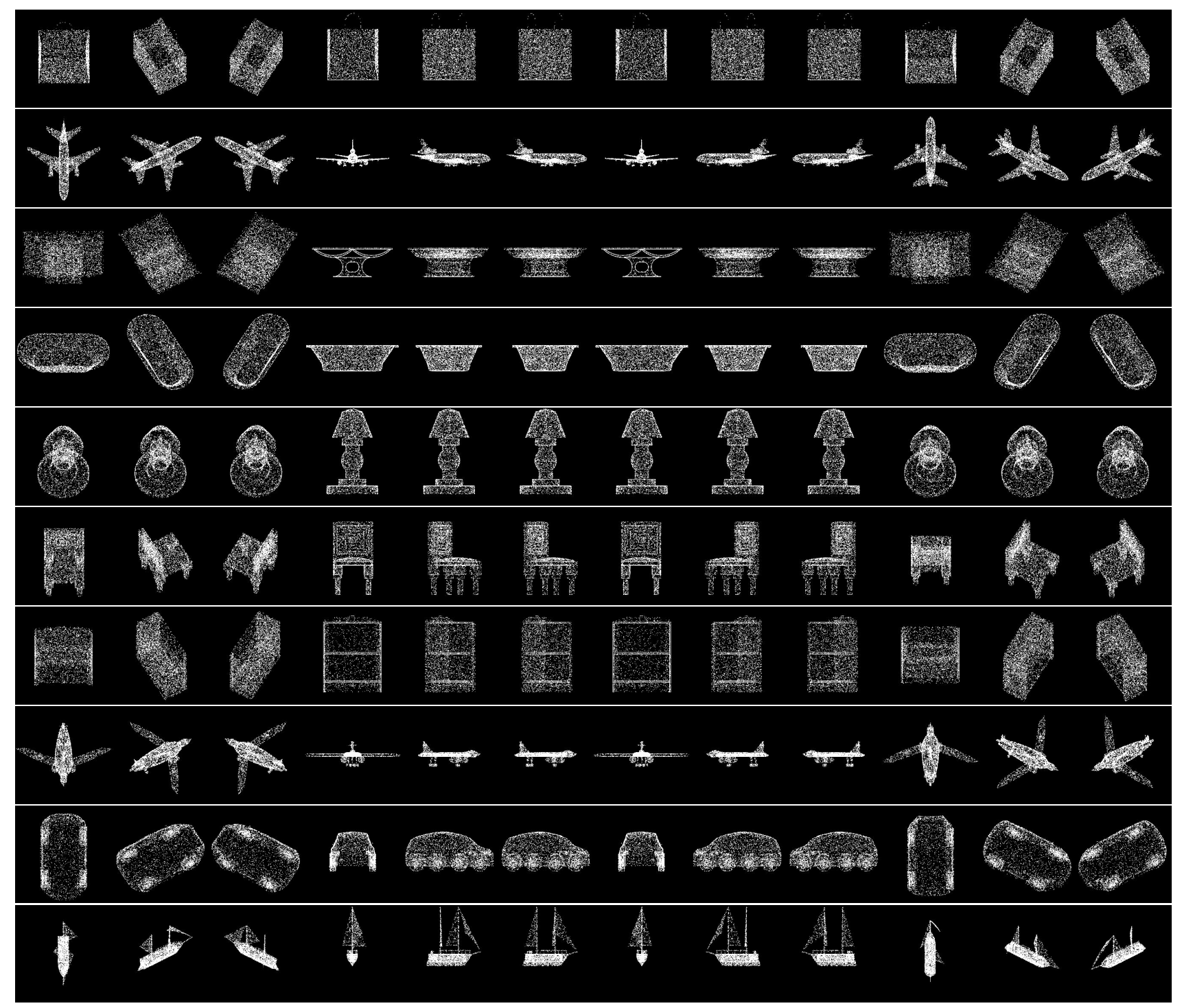

Figure 9. ShapeNet Core55. We show some examples of point cloud renderings of ShapeNet Core55 [8] used in training MVTN. Note how point cloud renderings offer more information about content hidden from the camera view-point (e.g. car wheels from the occluded side), which can be useful for recognition. For this figure, 12 spherical views are shown for each 3D shape. 


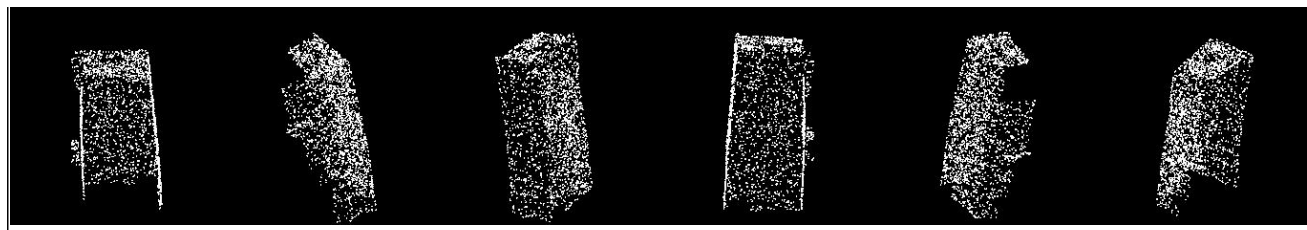

Object Only
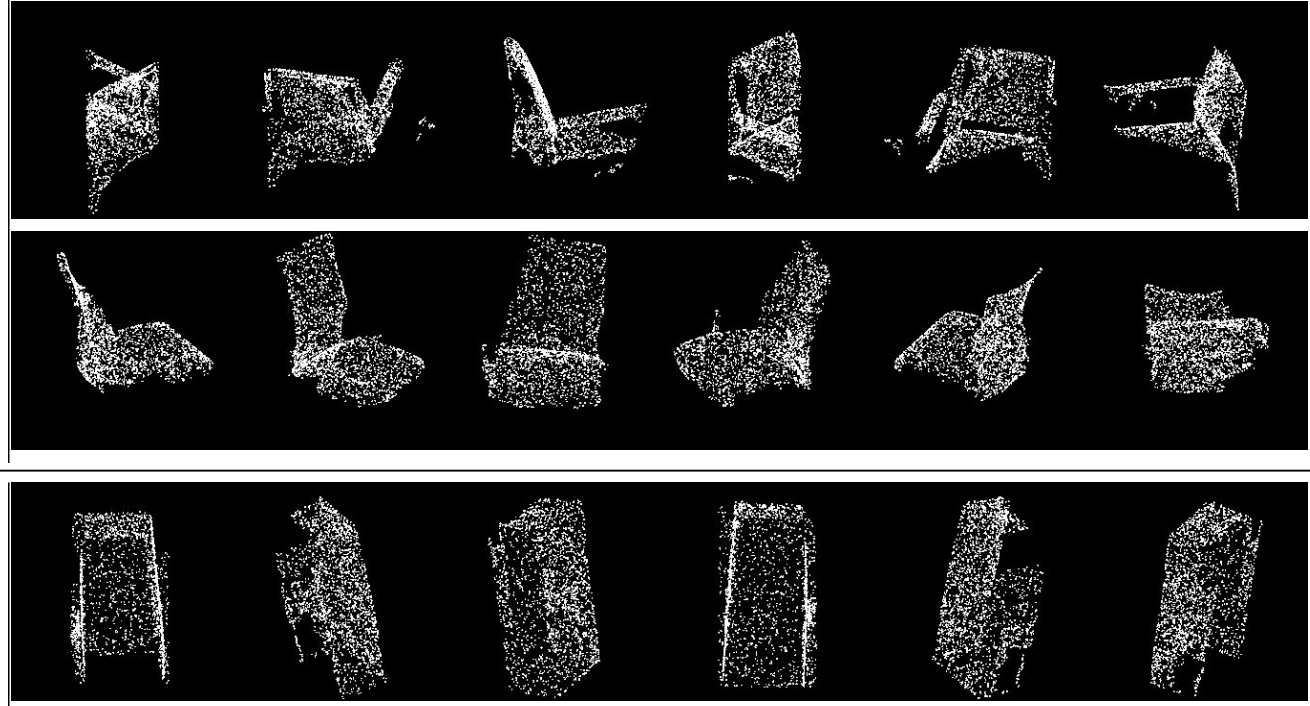

\section{With Background}
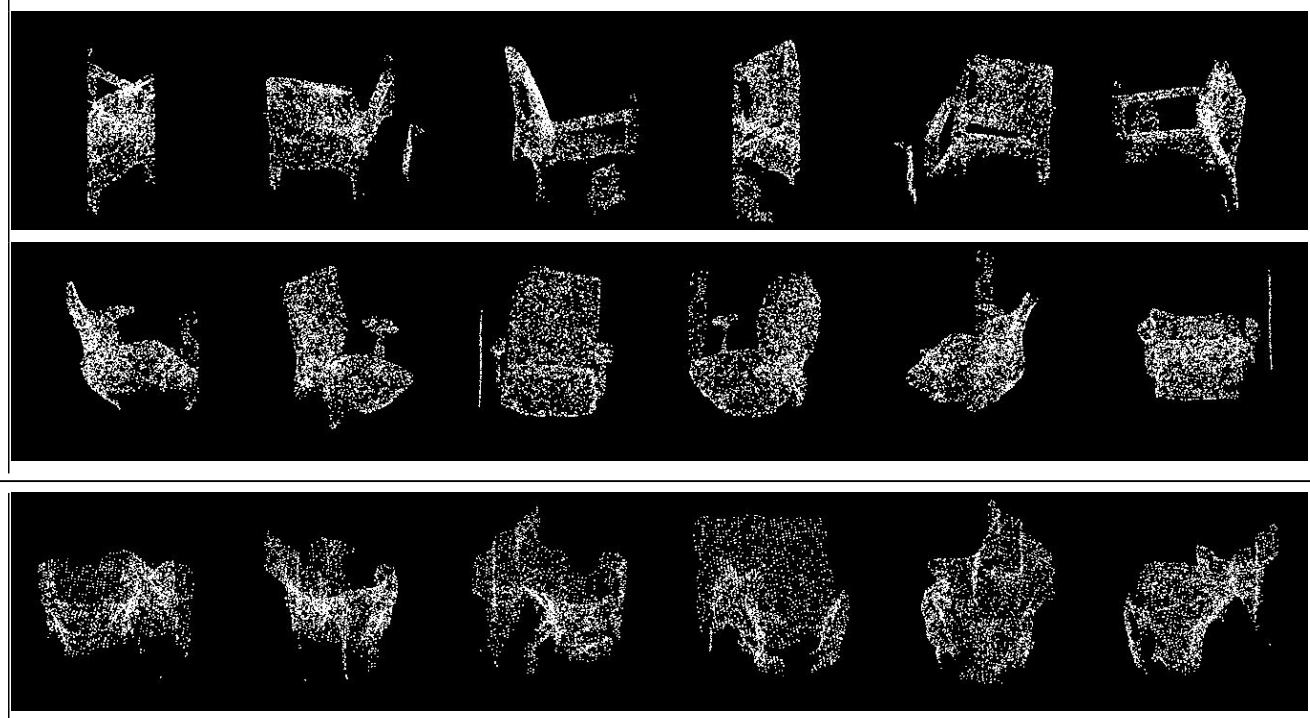

PB_T50_RS (Hardest)
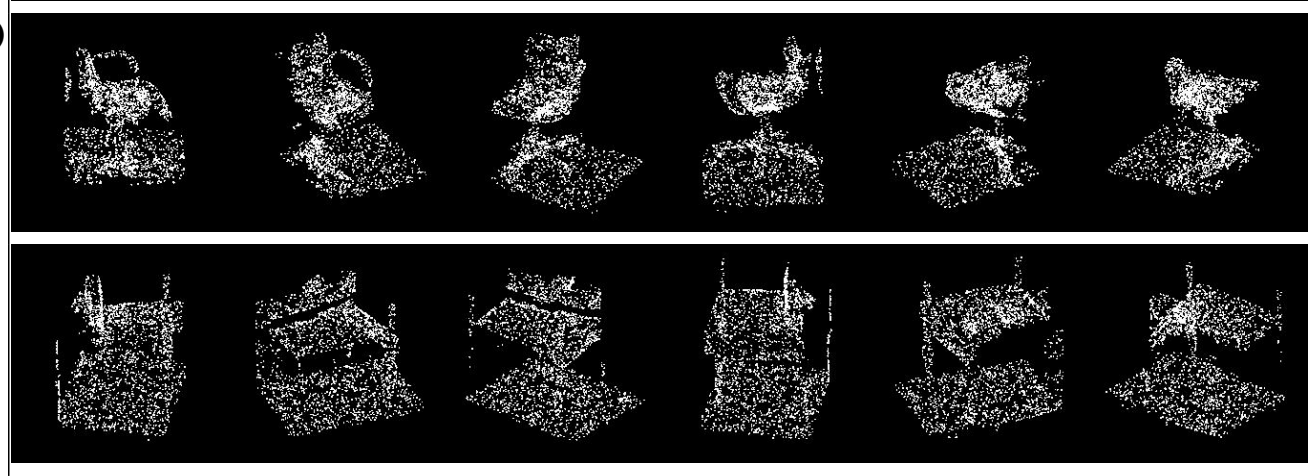

Figure 10. ScanObjectNN Variants. We show examples of point cloud renderings of different variants of the ScanObjectNN [72] point cloud dataset used to train MVTN. The variants are: object only, object with background, and the hardest perturbed variant (with rotation and translation). For this figure, six circular views are shown for each 3D shape. 


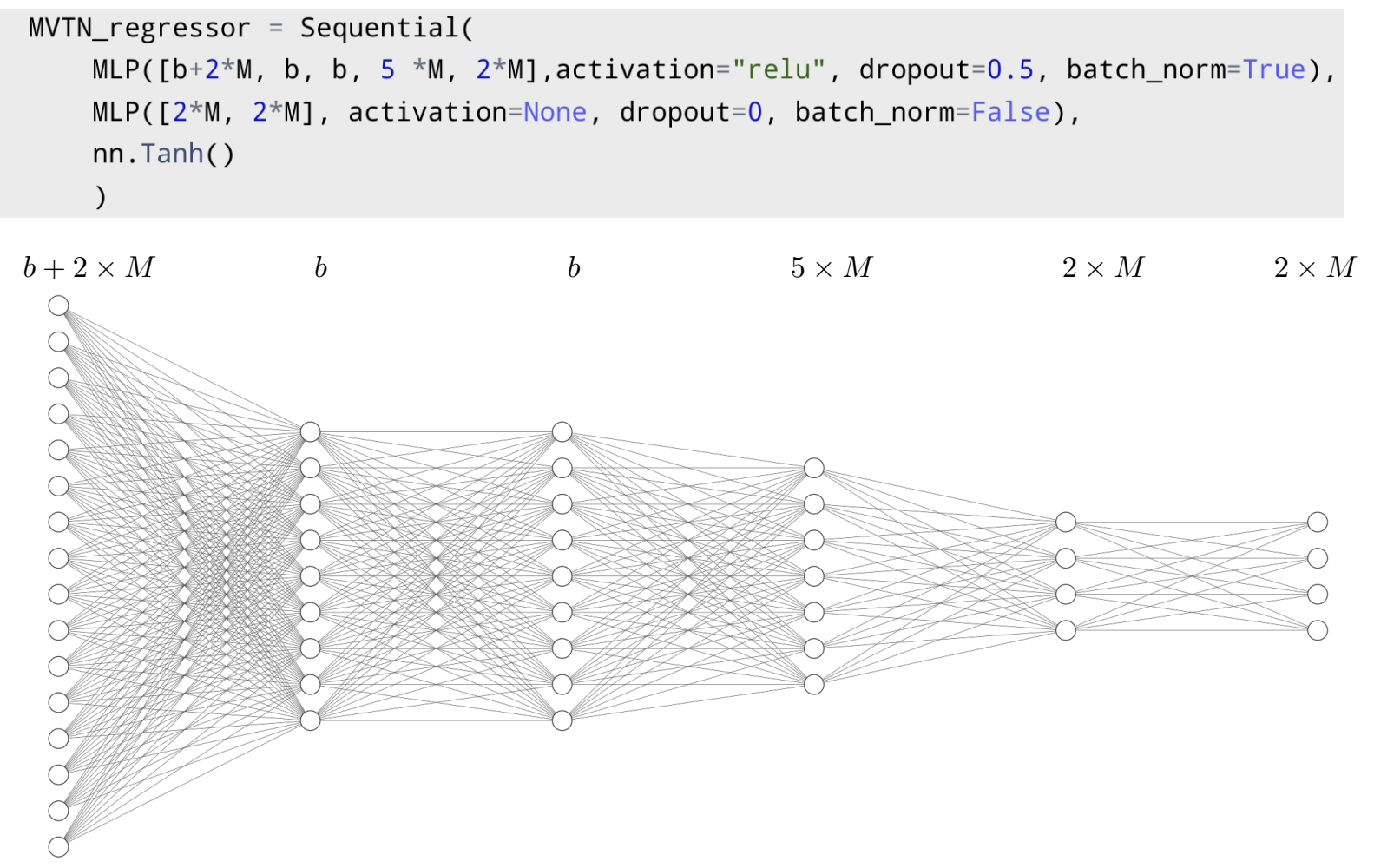

Figure 11. MVTN Network Architecture. We show a schematic and a code snippet for MVTN-spherical/MVTN-circular regression architectures used, where $b$ is the size of the point features extracted by the point encoder of MVTN and $M$ is the number of views learned. In most of our experiments, $b=40$, while the output is the azimuth and elevation angles for all the $M$ views used. The network is drawn using [48]

(a)

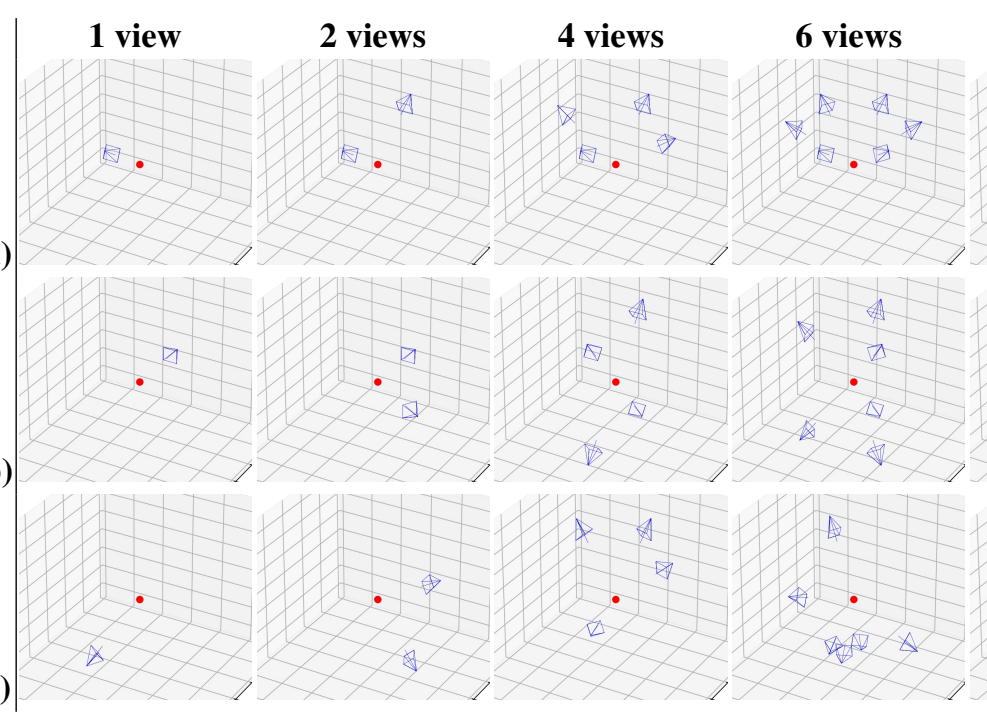

8 views 10 views
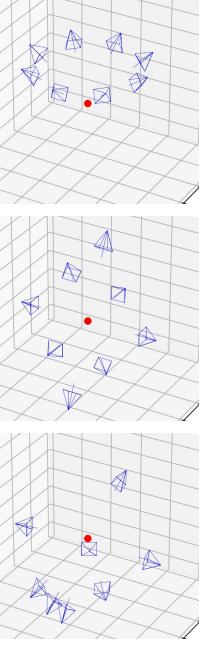

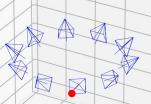

\section{2 views}
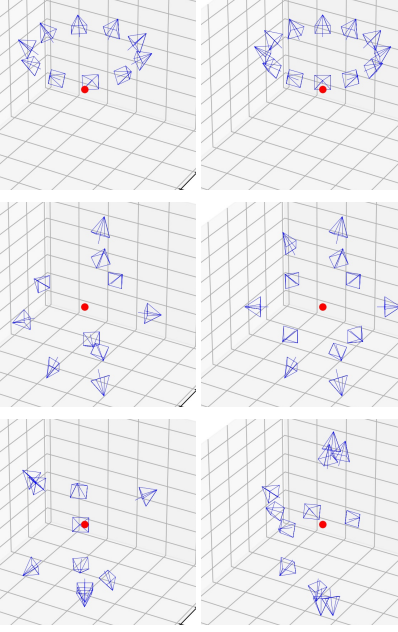

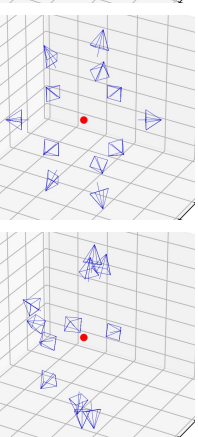

Figure 12. Views Configurations. We show some possible views configurations that can be used with a varying number of views. (a): circular, (b): spherical, (c): random 


\section{B. Additional Results}

\section{B.1. Classification and Retrieval Benchmarks}

We provide in Tables 9,10, and 11 comprehensive benchmarks of 3D classifications and 3D shape retrieval methods on ModelNet40 [79], ScanObjectNN [72], and ShapeNet Core55 [8, 68]. These tables include methods that use points as representations as well as other modalities like multi-view and volumetric representations. Our reported results of four runs are presented in each table as "max (avg \pm std)". Note in Table 9 how our MVTN improves the previous state-ofthe-art in classification (ViewGCN [75]) when tested on the same setup. Our implementations (highlighted using *) slightly differ from the reported results in their original paper. This can be attributed to the specific differentiable renderer of Pytorch3D [66] that we are using, which might not have the same quality of the non-differentiable OpenGL renderings [77] used in their setups.

\section{B.2. Rotation Robustness}

A common practice in the literature in 3D shape classification is to test the robustness of models trained on the aligned dataset by injecting perturbations during test time [55]. We follow the same setup as [55] by introducing random rotations during test time around the Y-axis (gravity-axis). We also investigate the effect of varying rotation perturbations on the accuracy of circular MVCNN when $M=6$ and $M=12$. We note from Fig. 13 that using less views leads to higher sensitivity to rotations in general. Furthermore, we note that our MVTN helps in stabilizing the performance on increasing thresholds of rotation perturbations.

\section{B.3. Occlusion Robustness}

To quantify the occlusion effect due to the viewing angle of the 3D sensor in our setup of 3D classification, we simulate realistic occlusion by cropping the object from canonical directions. We train PointNet [62], DGCNN [74], and MVTN on the ModelNet40 point cloud dataset. Then, at test time, we crop a portion of the object (from $0 \%$ occlusion ratio to $75 \%$ ) along the $\pm \mathrm{X}, \pm \mathrm{Y}$, and $\pm \mathrm{Z}$ directions independently. Fig. 15 shows examples of this occlusion effect with different occlusion ratios. We report the average test accuracy (on all the test set) of the six cropping directions for the baselines and MVTN in Fig. 14. Note how MVTN achieves high test accuracy even when large portions of the object are cropped. Interestingly, MVTN outperforms PointNet [62] by $13 \%$ in test accuracy when half of the object is occluded. This result is significant, given that PointNet is well-known for its robustness [62, 29].

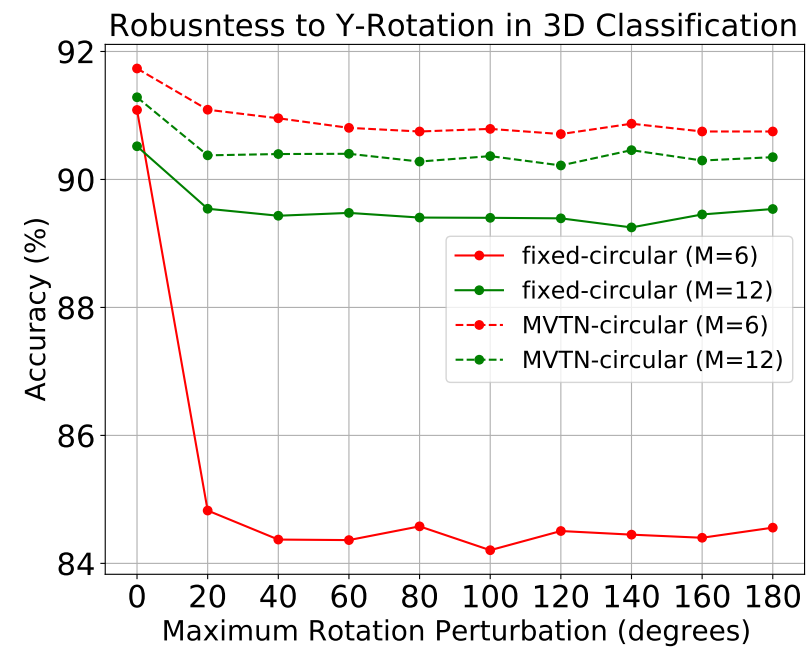

Figure 13. Robustness on a Varying Y-Rotation. We study the effect of varying the maximum rotation perturbation on the classification accuracies on ModelNet40. We compare the performance of circular MVCNN [69] to our circular-MVTN when it equips MVCNN when the number of views is 6 and 12. Note how MVTN stabilizes the drop in performance for larger Y-rotation perturbations, and the improvement is more significant for the smaller number of views $M$.

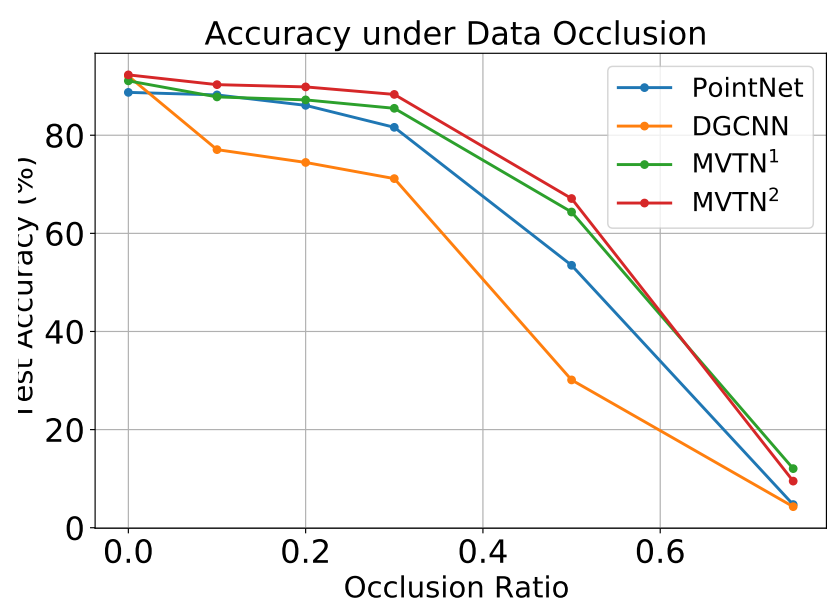

Figure 14. Occlusion Robustness of 3D Methods. We plot test accuracy $v s$. the Occlusion Ratio of the data to simulate the occlusion robustness of different 3D methods: PointNet [62], DGCNN [74], and MVTN. Our MVTN achieves close to $13 \%$ better than PointNet when half of the object is occluded. MVTN ${ }^{1}$ refers to MVTN with MVCNN as the multi-view network while MVTN $^{2}$ refers to MVTN with View-GCN as the multi-view network. 


\begin{tabular}{|c|c|c|c|}
\hline \multirow[b]{2}{*}{ Method } & \multirow[b]{2}{*}{ Data Type } & \multicolumn{2}{|c|}{ Classification Accuracy } \\
\hline & & (Per-Class) & (Overall) \\
\hline SPH [42] & Voxels & 68.2 & - \\
\hline LFD [11] & Voxels & 75.5 & - \\
\hline 3D ShapeNets [79] & Voxels & 77.3 & - \\
\hline VoxNet [59] & Voxels & 83.0 & 85.9 \\
\hline VRN [6] & Voxels & - & 91.3 \\
\hline MVCNN-MS [64] & Voxels & - & 91.4 \\
\hline FusionNet [33] & Voxels+MV & - & 90.8 \\
\hline PointNet [62] & Points & 86.2 & 89.2 \\
\hline PointNet++ [65] & Points & - & 91.9 \\
\hline KD-Network [43] & Points & 88.5 & 91.8 \\
\hline PointCNN [52] & Points & 88.1 & 91.8 \\
\hline DGCNN [74] & Points & 90.2 & 92.2 \\
\hline KPConv[71] & Points & - & 92.9 \\
\hline PVNet[83] & Points & - & 93.2 \\
\hline PTransformer[86] & Points & 90.6 & 93.7 \\
\hline MVCNN [69] & 12 Views & 90.1 & 90.1 \\
\hline GVCNN [20] & 12 Views & 90.7 & 93.1 \\
\hline ViewGCN [75] & 20 Views & 96.5 & 97.6 \\
\hline ViewGCN [75]* & 12 views & $90.7(90.5 \pm 0.2)$ & $93.0(92.8 \pm 0.1)$ \\
\hline ViewGCN [75]* & 20 views & $91.3(91.0 \pm 0.2)$ & $93.3(93.1 \pm 0.2)$ \\
\hline MVTN (ours)* & 12 Views & $92.0(91.2 \pm 0.6)$ & 93.8 $(93.4 \pm 0.3)$ \\
\hline MVTN (ours)* & 20 Views & $92.2(91.8 \pm 0.3)$ & $93.5(93.1 \pm 0.5)$ \\
\hline
\end{tabular}

Table 9. 3D Shape Classification on ModelNet40. We compare MVTN against other methods in 3D classification on ModelNet40 [79]. * indicates results from our rendering setup (differentiable pipeline), while other multi-view results are reported from pre-rendered views. Bold denotes the best result in its setup. In brackets, we report the average and standard deviation of four runs

\begin{tabular}{rccc}
\hline \multicolumn{1}{c}{ Method } & \multicolumn{3}{c}{ Classification Overall Accuracy } \\
Object with Background & Object Only & PB_T50_RS (Hardest) \\
\hline 3DMFV [3] & 68.2 & 73.8 & 63.0 \\
PointNet [62] & 73.3 & 79.2 & 68.0 \\
SpiderCNN [81] & 77.1 & 79.5 & 73.7 \\
PointNet ++ [65] & 82.3 & 84.3 & 77.9 \\
PointCNN [52] & 86.1 & 85.5 & 78.5 \\
DGCNN [74] & 82.8 & 86.2 & 78.1 \\
SimpleView [24] & - & - & 79.5 \\
BGA-DGCNN [72] & - & - & 79.7 \\
BGA-PN++ [72] & - & - & 80.2 \\
\hline ViewGCN & $91.9(91.12 \pm 0.5)$ & $90.4(89.7 \pm 0.5)$ & $80.5(80.2 \pm 0.4)$ \\
MVTN (ours) & $\mathbf{9 2 . 6}(92.5 \pm 0.2)$ & $\mathbf{9 2 . 3}(91.7 \pm 0.7)$ & $\mathbf{8 2 . 8}(81.8 \pm 0.7)$ \\
\hline
\end{tabular}

Table 10. 3D Point Cloud Classification on ScanObjectNN. We compare the performance of MVTN in 3D point cloud classification on three different variants of ScanObjectNN [72]. The variants include object with background, object only, and the hardest variant. ${ }^{*}$ indicates results from our rendering setup (differentiable pipeline), and we report the average and standard deviation of four runs in brackets. 


\begin{tabular}{rccc}
\hline & & \multicolumn{2}{c}{ Shape Retrieval (mAP) } \\
Method & Data Type & ModelNet40 & ShapeNet Core \\
\hline ZDFR [49] & Voxels & - & 19.9 \\
DLAN [21] & Voxels & - & 66.3 \\
SPH [42] & Voxels & 33.3 & - \\
LFD [11] & Voxels & 40.9 & - \\
3D ShapeNets [79] & Voxels & 49.2 & - \\
PVNet[83] & Points & 89.5 & - \\
MVCNN [69] & 12 Views & 80.2 & 73.5 \\
GIFT [2] & 20 Views & - & 64.0 \\
MVFusionNet [36] & 12 Views & - & 62.2 \\
ReVGG [68] & 20 Views & - & 74.9 \\
RotNet [40] & 20 Views & - & 78.4 \\
ViewGCN [75] & 20 Views & - & - \\
MLVCNN [37] & 24 Views & 92.2 & $\mathbf{8 2 . 9}(82.4 \pm 0.6)$ \\
\hline MVTN (ours) & 12 Views & $\mathbf{9 2 . 9}(92.4 \pm 0.6)$
\end{tabular}

Table 11. 3D Shape Retrieval. We benchmark the shape retrieval capability of MVTN on ModelNet40 [79] and ShapeNet Core55 [8, 68]. MVTN achieves the best retrieval performance among recent state-of-the-art methods on both datasets with only 12 views. In brackets, we report the average and standard deviation of four runs. 


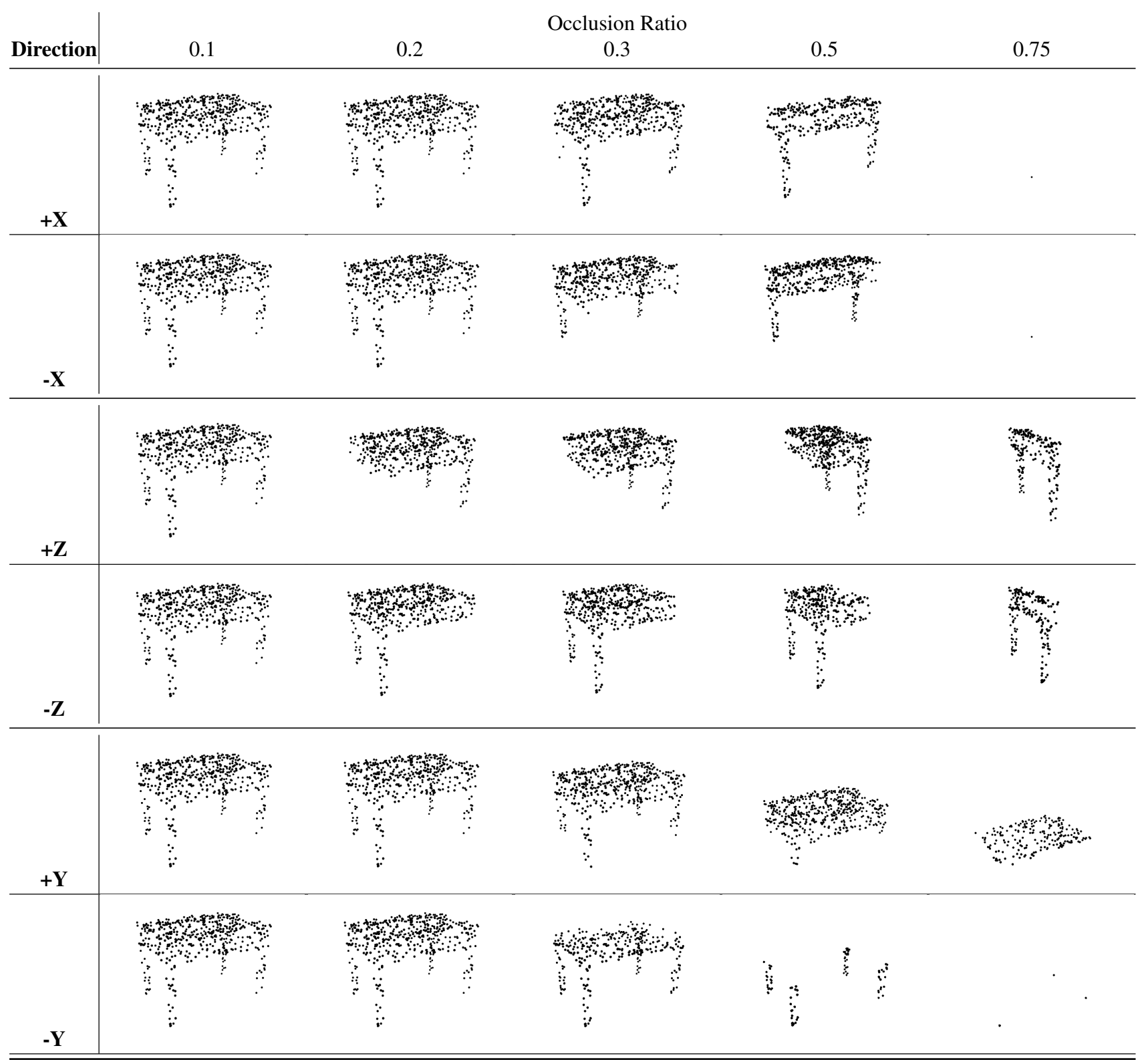

Figure 15. Occlusion of 3D Objects: We simulate realistic occlusion scenarios in 3D point clouds by cropping a percentage of the object along canonical directions. Here, we show an object occluded with different ratios and from different directions. 


\section{Analysis and Insights}

\section{C.1. Ablation Study}

This section introduces a comprehensive ablation study on the different components of MVTN, and their effect on test accuracy on the standard ModelNet40 [79].

MVTN Variants. We study the effect of the number of views $M$ on the performance of different MVTN variants (direct, circular, spherical). The experiments are repeated four times, and the average test accuracies with confidence intervals are shown in Fig. 16. The plots show how learned MVTN-spherical achieves consistently superior performance across a different number of views. Also, note that MVTNdirect suffers from over-fitting when the number of views is larger than four (i.e. it gets perfect training accuracy but deteriorates in test accuracy). This can be explained by observing that the predicted view-points tend to be similar to each other for MVTN-direct when the number of views is large. The similarity in views leads the multi-view network to memorize the training but to suffer in testing.

Backbone. In the main manuscript (Table 6), we study MVTN with ViewGCN as the multi-view network. Here, we study the backbone effect on MVTN with MVCNN as the multi-view network and report all results in Table 14. The study includes the backbone choice, and the point encoder choice. Note that including more sophisticated backbones does not improve the accuracy

Late Fusion. In the MVTN pipeline, we use a point encoder and a multi-view network. One can argue that an easy way to combine them would be to fuse them later in the architecture. For example, PointNet [62] and MVCNN [69] can be max pooled together at the last layers and trained jointly. We train such a setup and compare it to MVTN. We observe that MVTN achieves $91.8 \%$ compared to $88.4 \%$ by late fusion. More results are reported in Table 14

Light Direction Effect. We study the effect of light's direction on the performance of multi-view networks. We note that picking a random light in training helps the network generalize to the test set. Please see Fig. 17 for the results on circular MVTN with MVCNN when comparing this strategy to fixed light from the top or from camera (relative). Note that we use relative light in test time to stabilize the performance.

Effect of Object Color. Our main experiments used random colors for the objects during training and fixed them to white in testing. We tried different coloring approaches, like using a fixed color during training and test. The results are illustrated in Table 12.

Image size and number of points. We study the effect of rendered image size and the number of points sampled in a 4-view MVTN trained on ModelNet40 and report the overall accuracies (averaged over four runs) as follows. For image sizes $160 \times 160,224 \times 224$, and $280 \times 280$, the results

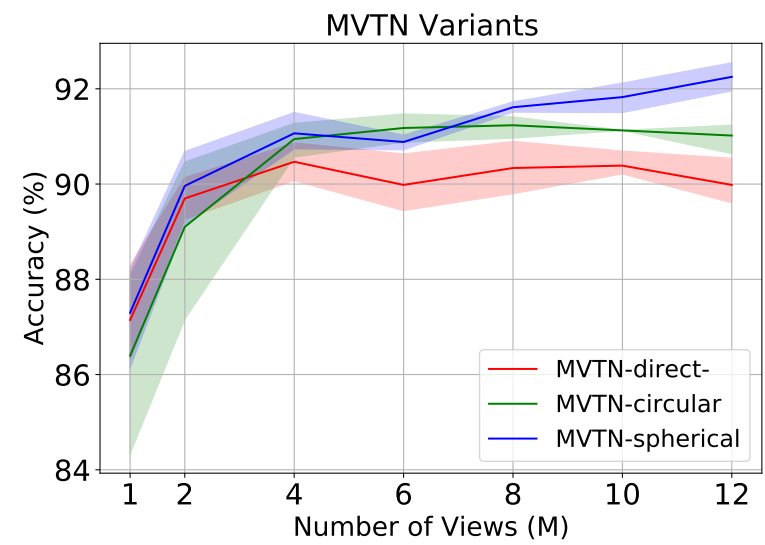

Figure 16. Variants of MVTN. We plot test accuracy vs. the number of views used in training different variants of our MVTN. Note how MVTN-spherical is generally more stable in achieving better performance on ModelNet40. 95\% confidence interval is also plotted on each setup (repeated four times).

are $91.0 \%, 91.6 \%$, and $91.9 \%$ respectively. For the number of randomly sampled points $P=512,1024$, and 2048, the results are $91.2 \% 91.6 \%$, and $91.6 \%$ respectively.

Learning Distance to the Object. One possible ablation to the MVTN is to learn the distance to the object. This feature should allow the cameras to get closer to details that might be important to the classifier to understand the object properly. However, we observe that MVTN generally performs worse or does not improve with this setup, and hence, we refrain from learning it. In all of our main experiments, we fixed the distance to 2.2 units, which is a good middle ground providing best accuracy. Please see Fig. 18 for the effect of picking a fixed distance in training spherical ViewGCN.

\section{C.2. Time and Memory of MVTN}

We compare the time and memory requirements of different parts of our pipeline to assess the MVTN module's contribution. We record FLOPs and MACs to count each module's operations and record the time of a forward pass for a single input sample and the number of parameters for each module. We find in Table 13 that MVTN contributes negligibly to the time and memory requirements of the multi-view networks and the 3D point encoders.

\section{C.3. Transferability of MVTN View-Points}

We hypothesize that the views learned by MVTN are transferable across multi-view classifiers. Looking at results in Fig. 20, 21, we believe MVTN picks the best views based on the actual shape and is less influenced by the multi-view network. This means that MVTN learns views that are more representative of the object, making it easier for any multiview network to recognize it. As such, we ask the following: can we transfer the views MVTN learns under one setting to 


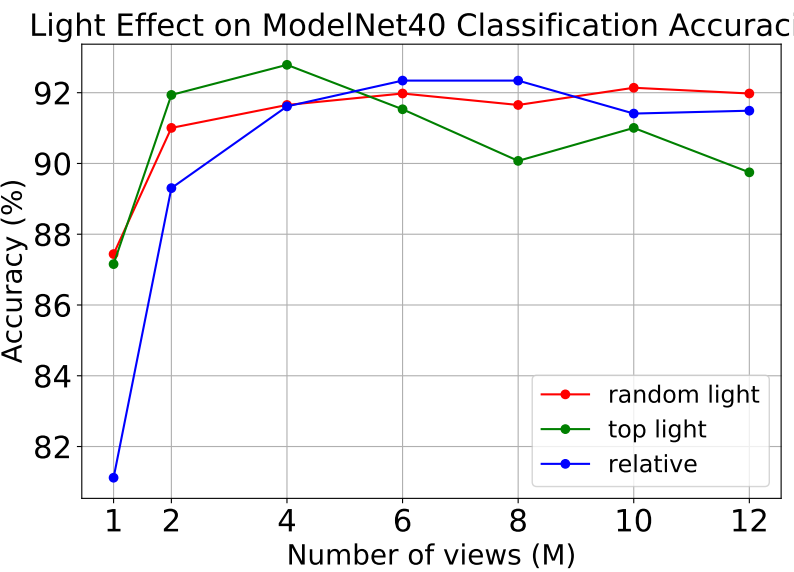

Figure 17. Light Direction Effect. We study the effect of light direction in the performance of the MVTN-circular. We note that randomizing the light direction in training reduce overfitting for larger number of views and leads to better generalization.

\begin{tabular}{c|cc}
\hline \multirow{2}{*}{ Method } & \multicolumn{2}{|c}{ Object } \\
& White & Randor \\
& Random \\
\hline Fixed views & $92.8 \pm 0.1$ & $92.8 \pm 0.1$ \\
MVTN (learned) & $93.3 \pm 0.1$ & $\mathbf{9 3 . 4} \pm 0.1$ \\
\hline
\end{tabular}

Table 12. Effect of Color Selection. We ablate selecting the color of the object in training our MVTN and when views are fixed in the spherical configuration. Fixed white color is compared to random colors in training. Note how randomizing the color helps in improving the test accuracy on ModelNet40 a little bit.

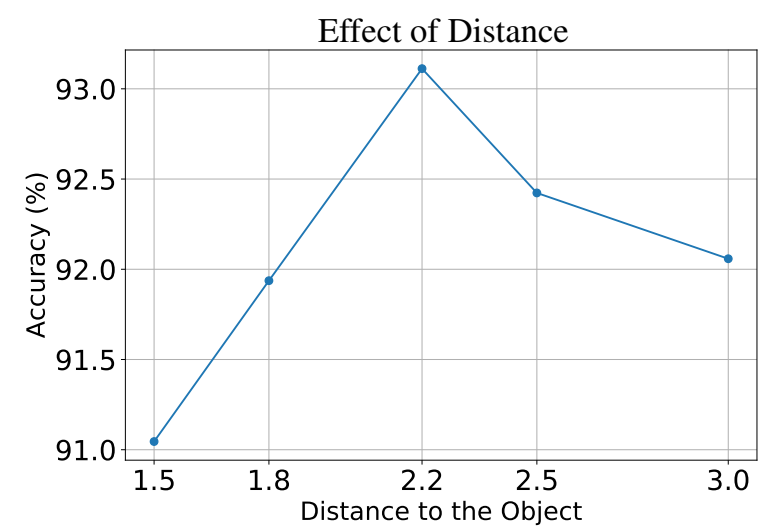

Figure 18. Effect of Distance to 3D Object. We study the effect of changing the distance on training a spherical ViewGCN. We show that the distance of 2.2 units to the center is in between far and close it and gives the best accuracy.

\section{a different multi-view network?}

To test our hypothesis, we take a 12-view MVTNspherical module trained with MVCNN as a multi-view network and transfer the predicted views to a ViewGCN multi-view network. In this case, we freeze the MVTN

\begin{tabular}{l|cccc}
\hline Network & FLOPs & MACs & Params. \# & Time \\
\hline PointNet & $1.78 \mathrm{G}$ & $0.89 \mathrm{G}$ & $3.49 \mathrm{M}$ & $3.34 \mathrm{~ms}$ \\
DGCNN & $10.42 \mathrm{G}$ & $5.21 \mathrm{G}$ & $0.95 \mathrm{M}$ & $16.35 \mathrm{~ms}$ \\
MVCNN & $43.72 \mathrm{G}$ & $21.86 \mathrm{G}$ & $11.20 \mathrm{M}$ & $39.89 \mathrm{~ms}$ \\
ViewGCN & $44.19 \mathrm{G}$ & $22.09 \mathrm{G}$ & $23.56 \mathrm{M}$ & $26.06 \mathrm{~ms}$ \\
\hline MVTN $^{*}$ & $\mathbf{1 8 . 5 2} \mathbf{K}$ & $\mathbf{9 . 2 6} \mathbf{K}$ & $\mathbf{9 . 0 9} \mathbf{K}$ & $\mathbf{0 . 9} \mathbf{~ m s}$ \\
MVTN $^{\circ}$ & $1.78 \mathrm{G}$ & $0.89 \mathrm{G}$ & $4.24 \mathrm{M}$ & $3.50 \mathrm{~ms}$ \\
\hline
\end{tabular}

Table 13. Time and Memory Requirements. We assess the contribution of the MVTN module to time and memory requirements in the multi-view pipeline. MVTN* refers to MVTN's regressor excluding the point encoder, while $\mathrm{MVTN}^{\circ}$ refers to the full MVTN module including PointNet as a point encoder.

module and only train ViewGCN on these learned but fixed views. ViewGCN with transferred MVTN views reaches $93.1 \%$ accuracy in classification. It corresponds to a boost of $0.7 \%$ from the $92.4 \%$ of the original ViewGCN. Although this result is lower than fully trained $\operatorname{MVTN}(-0.3 \%)$, we observe a decent transferability between both multi-view architectures.

\section{C.4. MVTN Predicted Views}

We visualize the distribution of predicted views by MVTN for specific classes in Fig. 19. This is done to ensure that MVTN is learning per-instance views and regressing the same views for the entire class (collapse scenario). We can see that the MVTN distribution of the views varies from one class to another, and the views themselves on the same class have some variance from one instance to another. We also show specific examples for predicted views in Fig. 20, 21. Here, we show both the predicted camera view-points and the renderings from these cameras. Note how MVTN shifts every view to better show the discriminative details about the $3 \mathrm{D}$ object. To test that these views are per-instance, we average all the views predicted by our 4-view MVTN for every class and test the trained MVCNN on these fixed per-class views. In this setup, MVTN achieves $90.6 \%$ on ModelNet 40 , as compared to $91.0 \%$ for the per-instance views and $89 \%$ for the fixed views.

\section{C.5. Shape Retrieval Examples}

We show qualitative examples of our retrieval results using the MVTN-spherical with ViewGCN in Fig. 22. Note that the top ten retrieved objects for all these queries are positive (from the same classes of the queries). 


\begin{tabular}{|c|c|c|c|c|c|c|c|c|c|}
\hline \multirow{2}{*}{$\begin{array}{c}\text { Views } \\
\text { number }\end{array}$} & \multicolumn{2}{|c|}{ Backbone } & \multicolumn{2}{|c|}{ Point Encoder } & \multicolumn{2}{|c|}{ Setup } & \multicolumn{2}{|c|}{ Fusion } & \multirow{2}{*}{$\begin{array}{c}\text { Results } \\
\text { accuracy }\end{array}$} \\
\hline & ResNet18 & ResNet50 & PointNet[62] & DGCNN[74] & circular & spherical & late & MVTN & \\
\hline 6 & $\checkmark$ & - & $\checkmark$ & - & $\checkmark$ & - & $\checkmark$ & - & $90.48 \%$ \\
\hline 6 & $\checkmark$ & - & $\checkmark$ & - & $\checkmark$ & - & - & $\checkmark$ & $91.13 \%$ \\
\hline 6 & $\checkmark$ & - & $\checkmark$ & - & - & $\checkmark$ & $\checkmark$ & - & $89.51 \%$ \\
\hline 6 & $\checkmark$ & - & $\checkmark$ & - & - & $\checkmark$ & - & $\checkmark$ & $91.94 \%$ \\
\hline 6 & $\checkmark$ & - & - & $\checkmark$ & $\checkmark$ & - & $\checkmark$ & - & $87.80 \%$ \\
\hline 6 & $\checkmark$ & - & - & $\checkmark$ & $\checkmark$ & - & - & $\checkmark$ & $91.49 \%$ \\
\hline 6 & $\checkmark$ & - & - & $\checkmark$ & - & $\checkmark$ & $\checkmark$ & - & $89.82 \%$ \\
\hline 6 & $\checkmark$ & - & - & $\checkmark$ & - & $\checkmark$ & - & $\checkmark$ & $91.29 \%$ \\
\hline 6 & - & $\checkmark$ & $\checkmark$ & - & $\checkmark$ & - & $\checkmark$ & - & $89.10 \%$ \\
\hline 6 & - & $\checkmark$ & $\checkmark$ & - & $\checkmark$ & - & - & $\checkmark$ & $90.40 \%$ \\
\hline 6 & - & $\checkmark$ & $\checkmark$ & - & - & $\checkmark$ & $\checkmark$ & - & $89.22 \%$ \\
\hline 6 & - & $\checkmark$ & $\checkmark$ & - & - & $\checkmark$ & - & $\checkmark$ & $90.76 \%$ \\
\hline 6 & - & $\checkmark$ & - & $\checkmark$ & $\checkmark$ & - & $\checkmark$ & - & $89.99 \%$ \\
\hline 6 & - & $\checkmark$ & - & $\checkmark$ & $\checkmark$ & - & - & $\checkmark$ & $89.91 \%$ \\
\hline 6 & - & $\checkmark$ & - & $\checkmark$ & - & $\checkmark$ & $\checkmark$ & - & $89.95 \%$ \\
\hline 6 & - & $\checkmark$ & - & $\checkmark$ & - & $\checkmark$ & - & $\checkmark$ & $90.43 \%$ \\
\hline 12 & $\checkmark$ & - & $\checkmark$ & - & $\checkmark$ & - & $\checkmark$ & - & $87.35 \%$ \\
\hline 12 & $\checkmark$ & - & $\checkmark$ & - & $\checkmark$ & - & - & $\checkmark$ & $90.68 \%$ \\
\hline 12 & $\checkmark$ & - & $\checkmark$ & - & - & $\checkmark$ & $\checkmark$ & - & $88.41 \%$ \\
\hline 12 & $\checkmark$ & - & $\checkmark$ & - & - & $\checkmark$ & - & $\checkmark$ & 91.82 \\
\hline 12 & $\checkmark$ & - & - & $\checkmark$ & $\checkmark$ & - & $\checkmark$ & - & $90.24 \%$ \\
\hline 12 & $\checkmark$ & - & - & $\checkmark$ & $\checkmark$ & - & - & $\checkmark$ & $90.28 \%$ \\
\hline 12 & $\checkmark$ & - & - & $\checkmark$ & - & $\checkmark$ & $\checkmark$ & - & $89.83 \%$ \\
\hline 12 & $\checkmark$ & - & - & $\checkmark$ & - & $\checkmark$ & - & $\checkmark$ & $91.98 \%$ \\
\hline 12 & - & $\checkmark$ & $\checkmark$ & - & $\checkmark$ & - & $\checkmark$ & - & $86.87 \%$ \\
\hline 12 & - & $\checkmark$ & $\checkmark$ & - & $\checkmark$ & - & - & $\checkmark$ & $88.86 \%$ \\
\hline 12 & - & $\checkmark$ & $\checkmark$ & - & - & $\checkmark$ & $\checkmark$ & - & $87.16 \%$ \\
\hline 12 & - & $\checkmark$ & $\checkmark$ & - & - & $\checkmark$ & - & $\checkmark$ & $88.41 \%$ \\
\hline 12 & - & $\checkmark$ & - & $\checkmark$ & $\checkmark$ & - & $\checkmark$ & - & $90.15 \%$ \\
\hline 12 & - & $\checkmark$ & - & $\checkmark$ & $\checkmark$ & - & - & $\checkmark$ & $88.37 \%$ \\
\hline 12 & - & $\checkmark$ & - & $\checkmark$ & - & $\checkmark$ & $\checkmark$ & - & $90.48 \%$ \\
\hline 12 & - & $\checkmark$ & - & $\checkmark$ & - & $\checkmark$ & - & $\checkmark$ & $89.63 \%$ \\
\hline
\end{tabular}

Table 14. Ablation Study. We study the effect of ablating different components of MVTN on the test accuracy on ModelNet40. Namely, we observe that using more complex backbone CNNs (like ResNet50 [31]) or a more complex features extractor (like DGCNN [74]) does not increase the performance significantly compared to ResNet18 and PointNet [62] respectively. Furthermore, combining the shape features extractor with the MVCNN [69] in late fusion does not work as well as MVTN with the same architectures. All the reported results are using MVCNN [69] as multi-view network. 


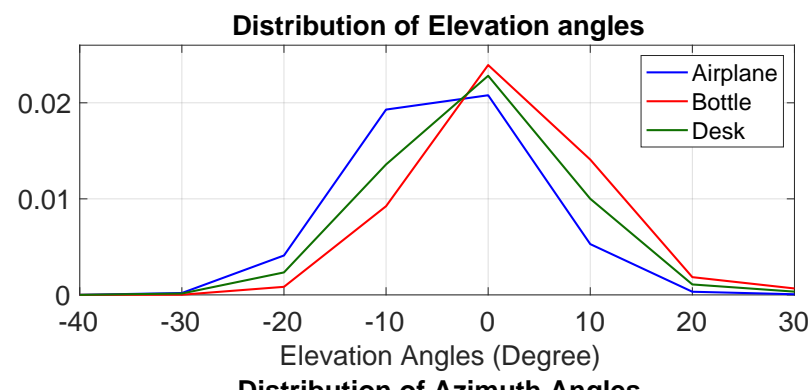

Distribution of Azimuth Angles

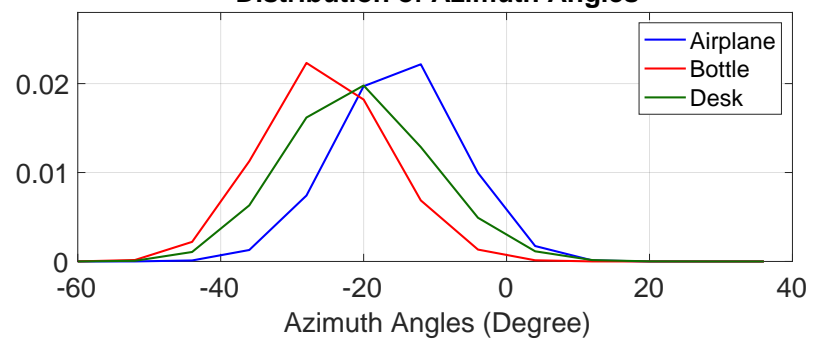

Figure 19. Visualizing MVTN learned Views. We visualize the distribution of azimuth and elevation angles predicted by the MVTN for three different classes. Note that MVTN learns interclass variations (between different classes) and intra-class variations (on the same class). 

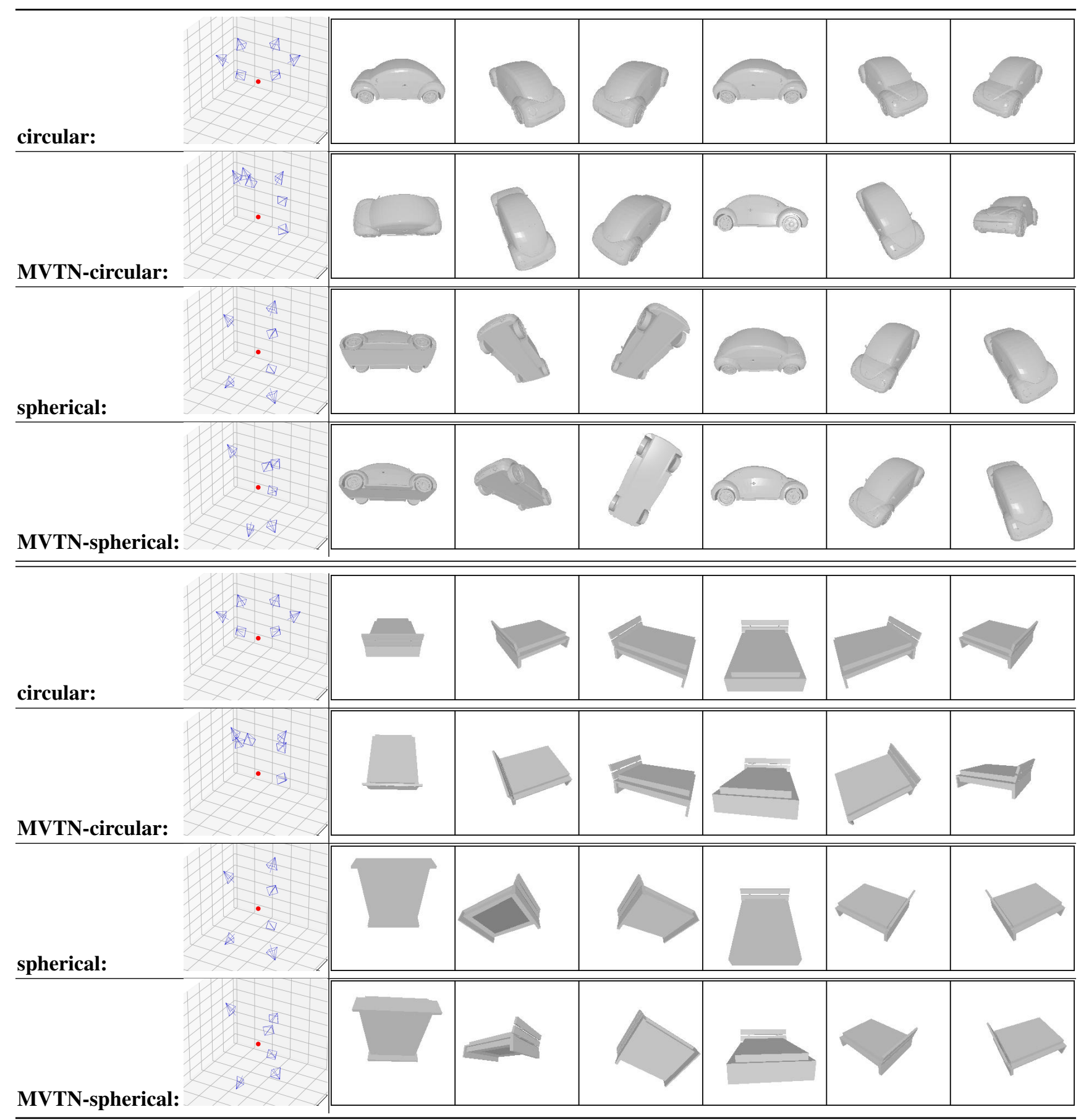

Figure 20. Qualitative Examples for MVTN predicted views (I): The view setups commonly followed in the multi-view literature are circular [69] or spherical [75, 40]. The red dot is the center of the object. MVTN-circular/MVTN-spherical are trained to predict the views as offsets to these common configurations. Note that MVTN adjust the original views to make the 3D object better represented by the multi-view images. 

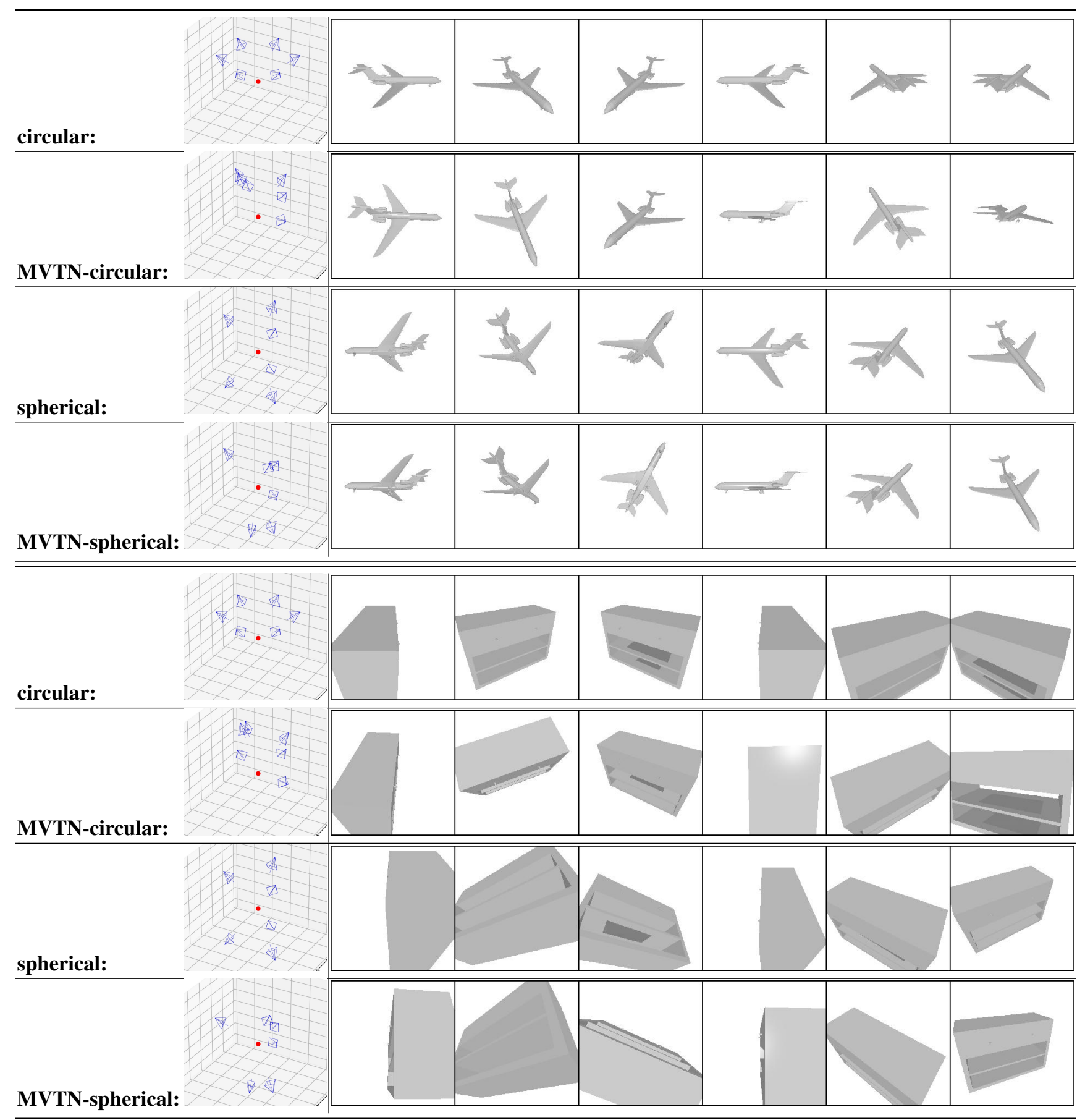

Figure 21. Qualitative Examples for MVTN predicted views (II): The view setups commonly followed in the multi-view literature are circular [69] or spherical [75, 40]. The red dot is the center of the object. MVTN-circular/MVTN-spherical are trained to predict the views as offsets to these common configurations. Note that MVTN adjust the original views to make the 3D object better represented by the multi-view images. 


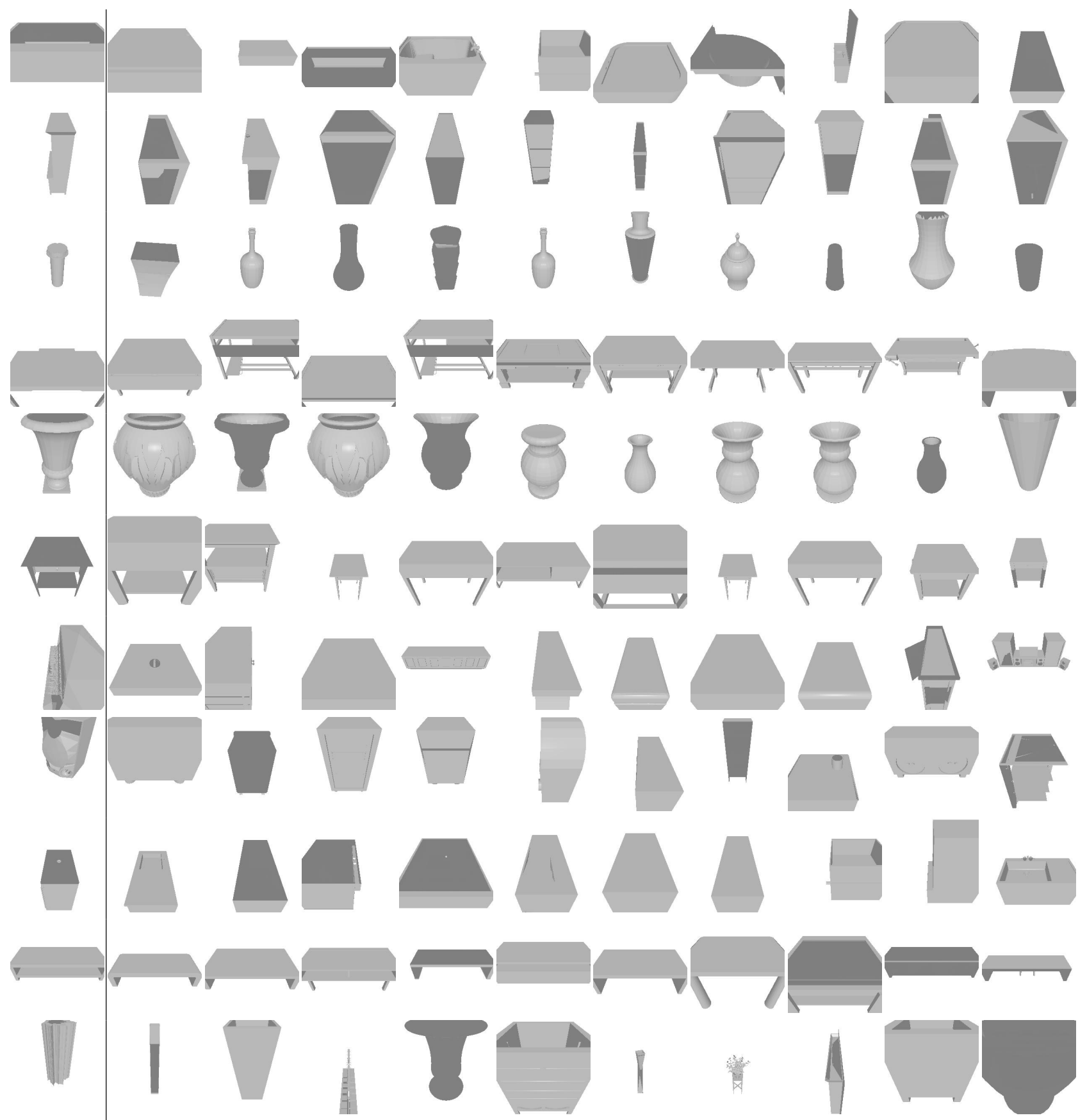

Figure 22. Qualitative Examples for Object Retrieval: (left): we show some query objects from the test set. (right): we show top ten retrieved objects by our MVTN from the training set. 